\title{
Flexible coding of object motion in multiple reference frames by parietal cortex neurons
}

\section{AUTHOR(S):}

Sasaki, Ryo; Anzai, Akiyuki; Angelaki, Dora E.; DeAngelis, Gregory C.

\section{CITATION:}

Sasaki, Ryo ... [et al]. Flexible coding of object motion in multiple reference frames by parietal cortex neurons. Nature Neuroscience 2020, 23(8): 1004-1015

\section{ISSUE DATE:}

2020-08-01

URL:

http://hdl.handle.net/2433/254045

\section{RIGHT:}

This is a post-peer-review, pre-copyedit version of an article published in 'Nature Neuroscience'. The final authenticated version is available online at: https://doi.org/10.1038/s41593-020-0656-0.; The full-text file will be made open to the public on 15 December 2020 in accordance with publisher's 'Terms and Conditions for Self-Archiving'.; この論文は出版 社版でありません。引用の際には出版社版をご確認ご利用ください。; This is not the published version. Please cite only the published version. 


\title{
Flexible coding of object motion in multiple reference frames by parietal
} cortex neurons

*Current institution for R.S.: Department of Neuroscience, Graduate School of Medicine, Kyoto

17 University, Kyoto, Japan

\author{
Ryo Sasaki $^{1}{ }^{*}$, Akiyuki Anzai $^{1}$, Dora E. Angelaki ${ }^{2}$ and Gregory C. DeAngelis ${ }^{1}$ \\ ${ }^{1}$ Department of Brain and Cognitive Sciences, Center for Visual Science, University of \\ Rochester, Rochester, New York USA
}

${ }^{2}$ Center for Neural Science, New York University, New York, New York, USA

21 


\section{ABSTRACT}

23 Neurons represent spatial information in diverse reference frames, but it remains unclear

24 whether neural reference frames change with task demands and whether these changes can

25 account for behavior. We examined how neurons represent the direction of a moving object

26 during self-motion, while monkeys switched, from trial to trial, between reporting object

27 direction in head- and world-centered reference frames. Self-motion information is needed to

28 compute object motion in world coordinates, but should be ignored when judging object motion

29 in head coordinates. Neural responses in the ventral intraparietal area are modulated by the task

30 reference frame, such that population activity represents object direction in either reference

31 frame. In contrast, responses in the lateral portion of the medial superior temporal area primarily

32 represent object motion in head coordinates. Our findings demonstrate a neural representation of

33 object motion that changes with task requirements. 


\section{INTRODUCTION}

Sensory signals are encoded in modality-specific reference frames at the sensory periphery, such as an eye-centered reference frame for visual signals and a head-centered reference frame

39 for vestibular signals. In downstream brain areas, signals are often transformed into non-native

40 reference frames, including intermediate or mixed reference frames ${ }^{1-13}$, and it is generally

41 presumed that different reference frames are useful for guiding different behaviors.

Natural behavior is flexible, however, and may require the observer to interpret the same

43 sensory signals in different reference frames, depending on task context (e.g., a soccer player

44 might judge motion of the ball relative to their head or relative to the goal). Having neural

45 representations that flexibly adapt to task demands may thereby simplify sensorimotor

46 transformations. While task-switching has been studied extensively with behavior and

47 neuroimaging ${ }^{14,15}$, and single-neuron correlates of switching task sets have been reported ${ }^{16,17}$,

48 little is known about whether the reference frame of neural representations changes dynamically

49 when judgements are made in different reference frames. When the reference frame required for

50 a task changes, do neural representations change accordingly or are neural reference frames

$51 \quad$ fixed?

52 Perception of object motion during self-motion provides an attractive model system for

53 studying this issue. When an observer is stationary, there is a unique mapping of object motion in

54 the world to image motion on the retina. However, when a translating observer views the same

55 moving object, image motion also depends on self-motion (Fig. 1). To judge object motion

56 relative to their head (e.g., a soccer player who wants to head the ball), one can rely on retinal

57 image motion, which is equivalent to motion in a head-centered reference frame if the eyes do

58 not move. However, to judge object motion relative to the world (e.g., a soccer goalie trying to 
59 judge whether a shot will be on goal), one needs to compensate for the visual consequences of

60 self-motion. How neural circuits incorporate information about self-motion to represent object

61 motion in the world is not well understood ${ }^{18,19}$. Moreover, it is not known whether neural

62 representations are dynamically updated to represent object motion in head or world coordinates

63 based on task instructions.

64 Numerous psychophysical studies have examined how perception of object motion discounts

65 image motion caused by self-motion, and have identified visual mechanisms that attempt to

66 isolate image motion produced by independent object motion ${ }^{20-24}$. Vestibular signals also aid in

67 dissociating the components of retinal image motion due to object motion and self-motion ${ }^{24-26}$.

68 Thus, we hypothesize that vestibular input may contribute to generating flexible representations

69 of object motion that update with task reference frame.

70 We trained macaque monkeys to discriminate object direction in either a world-centered or a

71 head-centered reference frame. Monkeys successfully switched between reference frames,

72 randomly across trials, and their performance was enhanced when both visual and vestibular self-

73 motion signals were available. We recorded neural activity from the lateral subdivision of the

74 medial superior temporal (MSTl) area, which has been suggested to play a role in coding object

75 motion ${ }^{27,28}$. MSTl neurons combine retinal image motion with extra-retinal signals related to

76 eye and head rotation ${ }^{29}$, suggesting that MSTl might be a viable candidate for representing

77 direction of object motion in different reference frames. We also recorded neural activity from

78 the ventral intraparietal (VIP) area, which is well known for its roles in representing visual

79 motion, as well as for carrying multisensory representations of visual, vestibular, tactile, and

80 auditory signals in diverse reference frames ${ }^{2,4,11,30,31}$. Thus, VIP is a good candidate to flexibly

81 represent object motion in a head- or world-centered reference frame. 
We find that responses of individual VIP neurons are modulated by the task reference frame,

83 such that their tuning shifts toward world coordinates when the task requires a world-centered

84 reference frame. In contrast, MSTl neurons do not show this effect. At the population level,

85 linear decoding of VIP activity accounts nicely for behavioral effects, whereas decoding of MST1

86 activity does not. Strikingly, a single set of decoding weights can accurately classify object

87 direction in either head or world coordinates based on VIP activity, but not MSTl activity. The

88 time course of the reference frame transformation in VIP is delayed relative to onset of visual

89 responses. Together, our findings demonstrate that VIP flexibly represents object motion in

90 different reference frames depending on task instructions, with self-motion signals being

91 incorporated into the computation when needed. More generally, our results provide striking

92 evidence that the reference frames of neural representations can be highly dynamic, and that the

93 same neural populations can carry information in different reference frames from moment to

94 moment.

\section{RESULTS}

97 We trained two macaque monkeys to report whether an object moves upward and rightward

98 or upward and leftward during lateral self-motion (Fig. 2a). In the world coordinate task,

99 monkeys judged object motion relative to vertical in a world-centered reference frame; in the

100 head coordinate task, animals reported object motion relative to a head-centered vertical

101 reference. Thus, for some stimulus conditions, the same patterns of random-dot motion on the

102 retina could give rise to opposite perceptual reports in the two different reference frames. In each

103 trial, the color and shape of the fixation point instructed the reference frame to be used (Fig. 2b),

104 and the two reference frame conditions were randomly interleaved. Self-motion information was 
105 provided by optic flow of background dots or by a congruent combination of optic flow and

106 physical translation of the animal on a motion platform. A partial cube frame that faded out

107 during object motion helped to instruct the task reference frame (Fig. 2c). If monkeys could

108 switch perfectly between task reference frames, psychometric functions for the two directions of

109 self-motion should overlap in the world coordinate task (Fig. 2d). In contrast, for the head

110 coordinate task, psychometric functions for the two opposite directions of self-motion should be

111 shifted by a predictable amount $(\triangle \mathrm{PSE}=35.5 \mathrm{deg}$, see Methods).

113 Monkeys can switch between world- and head-centered reference frames while judging

\section{4 object motion}

115 To summarize behavior, we computed average psychometric functions across all recording 116 sessions from the two monkeys (Fig. 3a). In the absence of self-motion (Object Only condition),

117 monkeys reported object direction with very little bias (Fig. 3a, black curve). During self-motion,

118 monkeys also performed quite accurately in the head coordinate task, as evidenced by

119 psychometric functions that were shifted by approximately the predicted amount (Fig. 3a, blue

120 and cyan curves). In the world coordinate task, psychometric functions for the two directions of

121 self-motion showed much smaller shifts, indicating that animals largely compensated for the

122 effect of their self-motion on object motion (Fig. 3a, magenta and brown curves). In the

123 Object+Combined condition, this compensation was nearly complete (magenta), whereas

124 compensation was substantially less complete in the Object+Visual condition (brown). This

125 finding demonstrates that vestibular signals enhance the monkeys' ability to judge object

126 direction in a world-centered reference frame. This effect presumably arises because adding

127 vestibular signals provides a more accurate and precise estimate of self-motion velocity, rather 
128 than facilitating switching between reference frames per se. However, we cannot differentiate 129 these possibilities.

130 To quantify these effects, we fit psychometric functions with cumulative Gaussian curves 131 and measured the point of subjective equality (PSE) for each self-motion direction, reference

132 frame, and recording session. Then, we computed the difference in PSE ( $\triangle \mathrm{PSE}$ ) between

133 leftward and rightward self-motion directions and compared the results between Object+Visual

134 and Object + Combined conditions (Fig. 3b). For the head coordinate task, mean $\triangle \mathrm{PSE}$ values for

135 both conditions (Fig. 3b, black triangle) are close to predicted values, although both are $10-15 \%$

136 greater than expected (two-tailed t-test, Object + Visual: $\mathrm{t}(184)=2.03, \mathrm{p}=0.044$;

137 Object + Combined: $\left.\mathrm{t}(184)=6.05, \mathrm{p}=8.0 \times 10^{-9}\right)$. Moreover, there is no substantive difference

138 between mean $\triangle$ PSE values for Object+Visual and Object+Combined conditions in the head

139 coordinate task (two-tailed paired t-test, $\mathrm{t}(368)=-0.71, \mathrm{p}=0.47$ ). This is expected because self-

140 motion information is not needed to perform the head coordinate task.

141 The pattern of results is strikingly different for the world coordinate task, where $\triangle \mathrm{PSE}$

142 values are much smaller. The mean $\triangle \mathrm{PSE}$ value in the Object+Visual condition is small (9.4 deg)

143 but consistently greater than zero $\left(\mathrm{t}(184)=13.9, \mathrm{p}=1.3 \times 10^{-50}\right)$, whereas the corresponding value

144 (0.75 deg) for the Object+Combined condition is not substantially different from zero (two-tailed

145 t-test, $\mathrm{t}(184)=1.34, \mathrm{p}=0.054)$. The difference in $\triangle \mathrm{PSE}$ values between Object+Visual and

146 Object+Combined conditions is quite robust across sessions in the world coordinate task (two-

147 tailed paired t-test, $\left.\mathrm{t}(368)=11.9, \mathrm{p}=1.7 \times 10^{-35}\right)$, unlike the head coordinate task. These behavioral

148 results demonstrate that monkeys successfully switch between world and head reference frames

149 from trial to trial, and that vestibular signals facilitate this transformation. 
Note that the slope of psychometric functions in Fig. 3a depends on the presence of self-

151 motion. Additional analysis showed that object motion discrimination thresholds are

152 significantly lower in the Object Only condition than in the conditions with self-motion, with

153 only modest differences between Object + Visual and Object + Combined conditions (Extended

154 Data Fig. 1).

155

\section{Effects of task reference frame on single-unit responses in VIP and MSTI}

We next investigated whether the activity of VIP and MSTl neurons was modulated by task

158 reference frame. We recorded from 223 VIP neurons and 177 MSTl neurons that met basic

159 inclusion criteria (see Methods). In general, responses of neurons in both areas are influenced by

160 both object motion and self-motion directions (Fig. 4, filled vs. open symbols). This is not

161 surprising since motion stimuli were transparent such that both object and self-motion vectors

162 impinged upon the receptive fields of the recorded populations (Extended Data Fig. 2). The key

163 question is whether responses depend on the instructed reference frame for the same object and

164 background motions, which can be assessed by comparing responses in the world coordinate task

165 with responses in the head coordinate task. Differences would suggest a representation of object

166 motion that changes with task demands. Indeed, the two example VIP neurons in Fig. 4a show

167 clear response differences between task reference frames in the Object + Combined condition

168 (magenta vs. cyan). In contrast, for the two example MSTl neurons in Fig. 4b, responses are

169 much more similar between the two task reference frames. These examples suggest that VIP

170 responses are more strongly modulated by task reference frame than MST1 responses. Data from

171 the Object+Visual condition for these same example neurons are shown in Fig. 4c,d; data from

172 additional example neurons are shown in Extended Data Fig. 3. 
To quantify response modulations related to task reference frame (without assuming a

174 functional form of those modulations), we first computed a modulation index (MI) that captures

175 the net response difference between head and world coordinate tasks (see Methods): larger

176 values of MI indicate greater response differences between task reference frames. We find that

177 MI values are significantly greater for VIP than MSTl neurons (Fig. 5a; Wilcoxon signed rank

178 test, Object+Visual condition: $\mathrm{Z}=4.10, \mathrm{p}=4.1 \times 10^{-5}$; Object + Combined: $\mathrm{Z}=4.46, \mathrm{p}=8.2 \times 10^{-6}$ ).

179 This suggests that the representation of object motion in VIP is more dependent on task

180 requirements than that in MST1.

181 To characterize the temporal dynamics of response modulations related to task reference

182 frame, we computed MI values within a $300 \mathrm{~ms}$ sliding window that was shifted in increments of

$18350 \mathrm{~ms}$. While MI values are greater in VIP than MSTl at almost all time points (Fig. 5b), they

184 grow substantially over time in both areas. Comparison of the time course of MI (Fig. 5b) with

185 the time course of population responses to the most effective object direction for each neuron

186 (Extended Data Fig. 4a) reveals that response modulations related to task reference frame arise

187 later than stimulus-driven modulations in both MST1 and VIP.

188 Differences in receptive field sizes and/or locations between brain areas could potentially

189 confound interpretation of the MI data. To evaluate this possibility, we performed an analysis of

190 covariance (ANCOVA) to test whether the difference in MI between MSTl and VIP was robust

191 to including receptive field size and eccentricity as covariates. We found a significant main

192 effect of brain area $(F(1,201)=5.0, p=0.026)$, with no significant dependence on receptive field

$193 \operatorname{size}(F(3,201)=0.7, p=0.55)$ or eccentricity $(F(3,201)=1.85, p=0.14)$.

194 While the MI data of Fig. 5a,b suggest greater task dependency of responses to object

195 motion in VIP, MI is sensitive to any differences in response between tasks, and does not 
196 necessarily reflect a shift in the neural representation toward world coordinates in the world task

197 condition. To assess this, we computed a (Pearson) correlation coefficient between tuning in the

198 Object Only condition and tuning in the conditions with self-motion. These correlations were

199 computed among tuning curves expressed in world coordinates (e.g., bottom row of Fig. 4), such

200 that alignment of the tuning curves in world coordinates would yield a large positive correlation

201 coefficient. This analysis was performed by pooling data across the Object+Visual and

202 Object + Combined conditions to gain statistical power, but results were very similar when

203 computed for the Object+Visual and Object+Combined conditions separately (not shown).

204 Across the populations of VIP and MSTd neurons, tuning correlations cover a broad range of

205 values (Fig. 5c), indicating that object tuning is not generally expressed in world coordinates.

206 Our data did not allow us to compute the correlation between tuning curves in head coordinates,

207 since there was insufficient overlap of object directions across self-motion directions when

208 expressed in head coordinates (e.g., top row of Fig. 4).

209 Critically, our design allowed us to test the hypothesis that correlation among tuning curves

210 in world coordinates becomes stronger in the world coordinate task as compared to the head

211 coordinate task. Indeed, for VIP, we find a robust increase in this correlation for the world

212 coordinate task (Fig. 5c, orange), consistent with a shift in neural tuning toward world

213 coordinates (Wilcoxon signed-rank test, $\mathrm{Z}=-4.45, \mathrm{p}=8.4 \times 10^{-6}$ ). The time course of this

214 correlation reveals that the shift toward world coordinates begins around 1000ms (Fig. 5d), just

215 as stimulus-driven responses in VIP are rising rapidly (Extended Data Fig. 4a). In contrast, we

216 find no significant dependence of tuning correlation on task reference frame for MSTl (Fig. 5c,

217 green, $\mathrm{Z}=0.175, \mathrm{p}=0.86$ ), with only a small shift toward world coordinates occurring late in the 
218 trial (Fig. 5d). These data suggest that VIP activity might account for the reference frame shifts

219 seen in behavior, whereas MSTl activity cannot.

221 Linear decoding of VIP population activity predicts behavioral performance across 222 reference frame conditions

To test whether the observed changes in single-unit responses with task reference frame

224 could account for behavioral performance, we used linear decoders (Fisher Linear Discriminant)

225 to classify object motion as rightward or leftward relative to vertical, based on responses of

226 pseudo-populations of 223 VIP neurons or 177 MSTl neurons (see Methods). We first

227 considered whether neural activity could account for behavioral performance if we trained

228 separate decoders to classify object motion direction in world or head coordinates (Fig. 6a). For

229 the head coordinate task, decoding either MSTl or VIP activity produced a pattern of results (Fig.

2306 b, blue and cyan curves) similar to the measured behavior (Fig. 3a, blue and cyan curves). This

231 implies that head-centered motion signals can be extracted from VIP and MSTl in the presence

232 of self-motion. Critically, the world coordinate task revealed clear differences in decoding

233 performance between brain areas. For MSTl, decoder performance curves (Fig. 6b right, brown

234 and magenta curves) shifted with self-motion direction to a much greater extent than seen in

235 behavior (Fig. 3a). In contrast, decoding of VIP responses (Fig. 6b left, brown and magenta

236 curves) reveals a pattern of results quite similar to behavior in the world coordinate task,

237 including substantially smaller shifts in the Object+Combined condition than the Object+Visual

238 condition. Because decoder weights were common across Object+Combined and Object+Visual

239 conditions, this improvement in decoder performance with inclusion of vestibular signals is not 240 guaranteed. 
242 psychometric functions exactly as done for behavioral data. Results for the VIP decoder lie fairly

243 close to behavioral performance for both world and head coordinate tasks (Fig. 6c, orange vs.

244 black symbols). In contrast, $\triangle \mathrm{PSE}$ values for the MSTl decoder differ greatly from behavioral

245 metrics in the world coordinate task (Fig. 6c, green vs. black symbols). These findings

246 demonstrate that MST1 responses only partially integrate self-motion signals and cannot be

247 effectively decoded in world coordinates, whereas the representation of object motion in VIP can

248 be decoded linearly to estimate object motion in either reference frame. Very similar results

249 (Extended Data Fig. 5) were obtained using a logistic regression decoder ${ }^{32}$, and results were

250 consistent across animals (Extended Data Fig. 6a-d).

251 In the analysis described above, we computed separate decoders for head and world

252 coordinate tasks, effectively assuming that the brain can apply different read-out weights to the

253 two task contexts. We can further ask whether it is possible to find a single set of decoding

254 weights that allows object direction to be read out in either reference frame. For this purpose, we 255 computed a single decoder that classifies object direction across both task reference frames (Fig.

256 6d). Strikingly, we found that a single decoder of VIP activity predicts performance in both

257 reference frames that is similar to behavior, whereas a single decoder of MSTl activity fails

258 almost completely for the world coordinate task (Fig. 6e). This finding, which was also quite

259 consistent across animals (Extended Data Fig. 6e-h), suggests that self-motion signals are

260 incorporated into VIP activity when animals are instructed to perform the task in a world-

261 centered reference frame (see Discussion). These results provide strong evidence for a novel role

262 of VIP in constructing a flexible representation of object motion. 
A potential confound is that differential responses between the world and head coordinate

264 tasks might be driven by retinal motion of the partial cube frame, which is different between task

265 reference frames. The partial cube frame was generally kept outside of the receptive fields and

266 was faded out during motion of the object and background dots (Fig. 2c) to avoid this confound;

267 nevertheless, it is possible that this could account for some of the task-related response

268 modulations. To assess this possibility, we computed a measure of direction-selective response to

269 the partial cube frame (cube effect index, CEI, see Methods) for both world and head coordinate

270 tasks, focusing on the first $500 \mathrm{~ms}$ of the trial during which the partial cube is visible and moving

271 but background dots are still largely invisible. For both brain areas, we found very few cells that

272 showed significant directionally-selective responses to the partial cube frame (Extended Data Fig.

$2737 \mathrm{a}, \mathrm{b}, \mathrm{d}, \mathrm{e}$ ), with little difference in median CEI between world and head task conditions

274 (Wilcoxon signed rank test, VIP: $Z=1.62, p=0.10$; MSTl: $Z=-0.83, p=0.41$ ). Nevertheless, we

275 divided the neural populations in half based on the absolute difference in CEI, $|\Delta \mathrm{CEI}|$, between

276 world and head tasks (Extended Data Fig. 7c,f), and we performed population decoding for these

277 two subsets of neurons. We found no reliable differences in decoder accuracy between subset of

278 neurons with relatively small and large values of $|\Delta \mathrm{CEI}|$ (Extended Data Fig. 7g,h for separate

279 decoders, Extended Data Fig. 7i,j for single decoder), suggesting that the partial cube frame was

280 not responsible for differences in VIP responses between world and head task conditions.

\section{Temporal dynamics of reference frame transformations}

283 Since monkeys were trained to switch reference frames based on a visual cue, there might be

284 some delay in gating self-motion signals into the computation of object direction, especially if

285 monkeys rely on judging motion of the partial cube frame, in addition to the color of the fixation 
286 point. Thus, we examined the time course of decoder performance using a $300 \mathrm{~ms}$ sliding

287 window that was shifted in increments of $50 \mathrm{~ms}$. For this analysis, we used separate decoders for

288 head and world coordinate tasks to give each area the best chance of success. We computed the

289 time course of decoder performance separately for stimulus conditions in which the correct

290 answer is the same for both tasks ("response matched") and conditions in which the correct

291 answers are different for the two task reference frames ("response conflict", Fig. 7a). Decoder

292 performance on response conflict trials is of special interest, as it should reveal the clearest

293 differences in neural representations across task reference frames.

294 Indeed, time courses of decoder accuracy for response conflict trials revealed striking

295 differences between task reference frames and brain areas (Fig. 7b). For VIP on response

296 conflict trials, decoder accuracy rose $\sim 500 \mathrm{~ms}$ later for the world coordinate task than the head

297 coordinate task (Fig. 7b, left), roughly consistent with the time course of average MI values (Fig.

298 5b). This late rise in VIP decoder performance for the world task, starting around 1000ms, is too

299 early to be attributed to re-appearance of the partial cube frame, which reaches zero luminance at

$300 \sim 1120 \mathrm{~ms}$ and does not become clearly visible for another few hundred ms. In contrast, for MST1,

301 decoder accuracy never reached much above chance for response conflict trials in the world

302 coordinate task, whereas accuracy rose quickly to high levels in the head coordinate task (Fig. 7b,

303 right). Thus, VIP responses undergo a delayed transformation that represents object motion in

304 world coordinates, whereas MSTl responses do not reliably represent motion in world

305 coordinates at any time. Note that decoder performance for VIP in the world task initially dips

306 below chance (0.5) levels, before rising precipitously (Fig. 7b, left). This below-chance

307 performance on response conflict trials is consistent with an early representation of object

308 direction in head coordinates in VIP, which later transitions to world coordinates. Below chance 
309 performance for response conflict trials is also seen for MSTl during much of the stimulus period,

310 again reflecting a representation of object direction in head coordinates (Fig. 7b, right).

311 In response matched conditions, the time course of decoder performance is largely similar

312 for MSTl and VIP in both head and world coordinate tasks (Fig. 7c). The only notable difference

313 is that MSTl decoder accuracy drops off somewhat toward the end of the stimulus period,

314 whereas accuracy of the VIP decoder is more sustained. This difference was not attributable to

315 receptive field coverage (Extended Data Fig. 2) or to the temporal profiles of neural responses

316 (Extended Data Fig. 4). We also examined decoder performance separately for trials that

317 followed a switch between task reference frames, as compared to trials for which the reference

318 frame did not change, and we found no clear differences (data not shown).

319 Our findings suggest that self-motion signals are incorporated into the computation of object

320 motion in VIP during the world coordinate task. To probe this idea further, we took advantage of

321 the fact that monkeys judged object direction in both reference frames for each unique random-

322 dot stimulus. This allowed us to perform cross-task decoding by training a classifier to perform

323 the world coordinate task using neural responses from the head coordinate task, or vice-versa.

324 For this analysis, we focused on the response conflict conditions, for which differences between

325 areas and task reference frames are most clear.

326 For VIP, the decoder could perform the head coordinate task using responses from the world

327 coordinate task (Fig. 8b, gray/black), but could not perform the world coordinate task using

328 responses from the head coordinate task (Fig. 8a, gray/black). This suggests that VIP activity

329 contains information about object motion in head coordinates in both task conditions, but only

330 represents object motion in world coordinates when the animal is performing the world

331 coordinate task. By comparison, a decoder of MSTl activity fails to perform the world 
332 coordinate task at all times using responses from either task condition (Fig. 8c), whereas it

333 accurately reports object direction in head coordinates when trained on responses from either

334 task condition (Fig. 8d). These observations are consistent with the hypothesis that both areas

335 carry robust information about object direction in a head-centered reference frame under all

336 conditions, whereas self-motion signals are incorporated into the computation of object motion

337 in VIP (but not MSTl) when the task requires a world-centered reference frame.

\section{Dissociation of choice signals from task reference frame signals}

$340 \quad$ Given that monkeys' choices are clearly different between the world and head task

341 conditions (Fig. 3), can the effects of task instruction on VIP responses be simply accounted for

342 by choice-related modulations? This question is especially relevant given that VIP neurons often

343 have strong choice-related activity that is not predictable from their stimulus tuning ${ }^{33}$. We

344 performed analyses, at both the single neuron and population levels, which demonstrate that

345 response modulations related to task instruction are distinct from choice-related activity.

346 At the single-unit level, we separately quantified choice- and task-related activity (see

347 Methods). For choice-related activity, we computed the familiar choice probability (CP) metric ${ }^{34}$,

348 which involves sorting responses into choice groups separately for each distinct stimulus and

349 task condition. Analogously, we quantified task-related modulations by computing a 'task

350 probability (TP)' metric, which involves sorting responses into groups based on task reference

351 frame. Critically, this is done separately for each choice and stimulus condition before z-scoring

352 and pooling, to ensure that TP is not influenced by choice. Many neurons in VIP and MST1 show

353 significant CP and/or TP values (Extended Data Fig. 8a,b); however, we find no correlation

354 between CP and TP across the population (VIP: $r=-0.062, p=0.36 ;$ MSTl: $r=-0.041, p=0.59$, 
355 Pearson correlation). To probe this dissociation further, we computed TP separately for left and

356 right choices (Extended Data Fig. 8c,d), and we find these values to be strongly correlated (VIP:

$357 \mathrm{r}=0.76, \mathrm{p}=9.9 \times 10^{-42} ; \mathrm{MSTl}: \mathrm{r}=0.76, \mathrm{p}=1.6 \times 10^{-32}$, Pearson correlation), indicating that task-

358 related modulations for individual neurons are consistent across left and right choices. Similarly,

359 we find that $\mathrm{CP}$ values are correlated across task reference frames (VIP: $\mathrm{r}=0.50, \mathrm{p}=2.3 \times 10^{-15}$;

360 MSTl: $r=0.33, p=1.7 \times 10^{-5}$, Extended Data Fig. 8e,f, Pearson correlation). Together, these

361 results show that choice and task reference frame have separable effects on responses of

362 individual neurons.

363 To assess whether performance of our decoders could be confounded with choice-related

364 activity, we devised an approach (see Methods) to largely remove either the choice-related or

365 task-related response modulations for each neuron. Our approach for removing choice-related

366 activity virtually eliminated significant CP values while leaving TP values largely unchanged

367 (compare Extended Data Fig. 9a,b with Extended Data Fig. 8a,b). Similarly, our method for

368 removing task-related modulations largely eliminated significant TP values while leaving CP

369 values largely unchanged (Extended Data Fig. 9c,d vs. Extended Data Fig. 8a,b). Critically,

370 decoder performance on response conflict trials was greatly impaired when task-related

371 modulations were removed (compare Extended Data Fig. 9e to Fig. 7b), whereas there was little

372 effect on decoder performance of removing choice-related modulations in response (Extended

373 Data Fig. 9f). These findings demonstrate that the flexible reference frame exhibited by VIP

374 activity cannot simply be attributed to choice-related activity.

375

376 DISCUSSION 
By training monkeys to report object motion in either head or world coordinates, we

378 examined whether neurons represent object direction in a fixed reference frame or one that

379 changes with task requirements. Our findings demonstrate that VIP, but not MSTl, contains a

380 flexible representation of object motion that dynamically changes from moment to moment to

381 represent object direction relative to the head or world. The dynamics of these effects suggest

382 that self-motion signals are incorporated into the representation of object motion in VIP when the 383 task requires a world-centered representation.

384 Our findings provide an important advance in understanding how the brain represents

385 object motion during self-motion, providing the first evidence for a flexible multi-sensory

386 representation that can signal object motion relative to the head or world. The fact that addition

387 of vestibular stimulation facilitates reference frame transitions is consistent with previous

388 psychophysical and neurophysiological studies showing that vestibular input helps to dissociate

389 object motion and self-motion ${ }^{19,24,25,35,36}$. More generally, our findings provide compelling

390 evidence that the reference frame of neural representations is not static, and can be powerfully

391 modulated by task instructions.

\section{Caveats and limitations}

It was difficult for animals to switch reference frames from trial to trial without the

395 partial cube frame, potentially because the screen edges provide a strong head-centered frame in

396 the absence of the partial cube (see Methods). Although the partial cube frame gradually became

397 invisible while the moving object became visible (Fig. 2c), it is possible that animals might have

398 tried to judge the horizontal velocity of object motion relative to specific edges or corners of the

399 partial cube, rather than judging object velocity relative to the world. Two factors argue strongly 
400 against this interpretation: 1) a visual strategy of judging object velocity relative to specific

401 features of the partial cube frame would not explain the behavioral and neural effects of physical

402 motion of the platform, which provided vestibular signals. 2) We performed control experiments

403 in which we varied the location of the partial cube frame in depth from trial to trial. If animals

404 were reporting object motion relative to the near or far edges of the cube frame, their $\Delta \mathrm{PSE}$

405 values would be expected to depend systematically on cube depth, since the retinal velocity of

406 cube features depends on their distance from the observer. In contrast, we found no significant

407 dependence on depth of the cube (Extended Data Fig. 10), suggesting that the animals did not

408 employ this strategy.

409 Because we were not able to record from large ensembles of neurons simultaneously, our

410 decoding analyses were based on pseudo-populations of neurons for which the noise correlations

411 were largely unknown (see Methods). Thus, our analyses effectively assumed that neurons had

412 independent noise, which is not accurate ${ }^{37,38}$. It is well established that correlated noise among

413 neurons can influence the information content (or sensitivity) of a population code ${ }^{39-41}$.

414 Importantly, all of our main conclusions are based upon estimates of biases in decoding

415 performance, not on sensitivity measures. While we cannot rule out the possibility that

416 correlated noise would influence biases in decoder performance, it seems unlikely that the

417 pattern of results would change qualitatively.

418 Our analyses assume that the monkeys always identified the object as moving relative to

419 the background field of dots, which is a safe assumption for two reasons. First, object motion

420 always contained a substantial vertical component that was not compatible with the horizontal

421 self-motion of the animal. Second, the moving object was visually distinct from background

422 dots (see Methods), such that it was easily segmented from the background. More generally, the 
423 brain has to solve a causal inference problem to discern whether the retinal motion of an object is

424 produced by self-motion or also reflects independent movement of the object relative to the

425 scene ${ }^{42}$. The neural basis of this causal inference process will be the topic of future studies.

427 Relationship to previous studies

A previous study ${ }^{29}$ reported that visual tracking neurons in area MSTl represent visual

429 target motion in world coordinates while macaques tracked a target using voluntary eye and head

430 rotations. It was suggested that MSTl neurons represent world-centered target motion by

431 combining retinal motion signals, efference copy signals related to smooth eye movement, and

432 vestibular signals related to head rotation. On the surface, the findings of Ilg et al. ${ }^{29}$ appear to

433 conflict with our finding that MSTl does not represent object motion in world coordinates.

434 However, our subjects performed the discrimination task while their eyes and head remained

435 oriented straight ahead. Whereas the tracking task used by Ilg et al. ${ }^{29}$ elicited extra-retinal

436 signals related to eye and head rotation, our stimuli involved real or simulated head translations.

437 The findings of Ilg et al. ${ }^{29}$ are therefore not incompatible with ours, and collectively they

438 suggest that some MSTl neurons may account for eye and head rotations, but that MSTl does not

439 contain a generalized representation of world-referenced object motion.

$440 \quad$ Reference frames of different sensory signals in area VIP have been the focus of several

441 previous studies. Facial tactile receptive fields (RFs) are coded in a head-centered reference

442 frame ${ }^{2}$, whereas auditory RFs are organized in a continuum between eye- and head-centered

443 coordinates ${ }^{10,11}$. Visual RFs and heading tuning (optic flow) are represented mainly in an eye-

444 centered reference frame ${ }^{11,31,43}$, although some studies have described head-centered visual RFs

445 in VIP as well ${ }^{4}$. In contrast, vestibular heading signals in VIP are coded in body- or world- 
446 centered reference frames ${ }^{30,44}$. One recent study showed that the reference frame of vestibular

447 heading tuning in VIP depended on whether gaze was focused on a head- or world-fixed target ${ }^{44}$,

448 consistent with the idea that VIP has flexible reference frames. However, our findings show that

449 VIP reference frames can change just by task instructions, and do not require different motor $450 \quad$ actions ${ }^{44}$.

451 Our findings substantially extend previous work on the context-dependence and

452 dynamics of spatial reference frames. Human neuroimaging studies have reported that visual

453 motion signals can be represented in retinal or head coordinates depending on the spatial

454 allocation of attention ${ }^{45}$, although this finding has been refuted by other studies ${ }^{46}$. Human fMRI

455 studies also demonstrated that activity in parietal and premotor cortex reflected different spatial

456 reference frames depending on the sensory modality used to specify target location ${ }^{47}$. Previous

457 studies have also demonstrated that the reference frame of neural activity in monkeys is dynamic,

458 changing over time relative to task events ${ }^{8,48}$. Our findings extend this work in two important

459 ways. First, we demonstrate that neural activity is modified by task instructions to represent

460 object motion in the reference frame required for each task condition. Second, we directly

461 compare neural and behavioral correlates of dynamically changing task reference frames,

462 allowing for a more direct assessment of whether changes in neural activity with task reference

463 frame can explain behavior. In contrast, previous neurophysiological studies of reference frames

464 have generally just varied the position of an effector without requiring animals to make a

465 perceptual report.

466 The Duncker illusion ${ }^{49,50}$ describes biases in the perceived trajectory of an object when it

467 moves relative to a moving background. The perceptual biases exhibited by our monkeys cannot

468 simply be accounted for by the Duncker illusion because the image motion of the target object 
469 and background dots are identical in the world and head coordinate tasks, yet the perceptual

470 reports are strikingly different (Fig. 3a).

472 Implications of flexible reference frames

473 The primary contribution of our study is to demonstrate that neural reference frames can

474 change dramatically based on task instructions. Secondarily, these results have implications for

475 understanding the variability of outcomes across previous studies of neural reference frames.

476 Two examples of such variability, as noted above, include the incidence of head-centered visual

477 receptive fields in VIP ${ }^{4,43}$ and the existence of spatiotopic representations in human visual

478 cortex ${ }^{45}{ }^{46}$. Given that the vast majority of neurophysiological studies of reference frames have

479 not used a behavioral task that enforces a specific task reference frame, findings could vary with

480 the intrinsic (and uncontrolled) reference frame that the animal employs, which in turn may

481 depend on the animal's previous experience or training history. Findings could also vary with the

482 stimuli used, which might bias the animal toward adopting a specific task reference frame.

483 We found that a single set of decoding weights could be used to classify object direction

484 in either head or world coordinates, based on VIP activity. This result could arise because task

485 instructions simply shift the 'population hill' of neural activity along the stimulus axis, similar to

486 changing object direction itself. A pure horizontal shift of the population hill, in which the

487 pattern of population activity is simply translated along the object direction axis, would occur if

488 all tuning curves for object direction simply shifted with self-motion in the world coordinate task.

489 This was clearly not the case based on inspection of tuning curves from individual neurons (Fig.

4904 and Extended Data Fig. 3), as well as the broad distribution of tuning correlation values in Fig.

$4915 \mathrm{c}$. We found that self-motion has diverse effects on object motion tuning in the world 
492 coordinate task, including shifts, gain changes, and changes in shape of tuning. Thus, it remains

493 an interesting topic for future studies to determine how a single decoder can estimate object

494 direction in head or world coordinates based on such diverse modulations at the single-unit level.

495

496 Acknowledgements: This work was supported by NIH grant EY01618 (to GCD), The Uehara

497 Memorial Foundation (to RS), the Japan Society for the Promotion of Science (to RS), and an

498 NEI CORE grant (EY001319). We thank D. Graf, S. Shimpi, and E. Murphy for excellent

499 technical support, and J. Wen and A. Yung for programming support.

500

501 Author contributions: R.S. and G.C.D. conceived and designed research; R.S. performed

502 experiments; R.S. analyzed data; A.A. built recording system; R.S., A.A., D.E.A., and G.C.D.

503 interpreted results of experiments; R.S. prepared figures; R.S. and G.C.D. drafted manuscript;

504 R.S., A.A., D.E.A., and G.C.D. edited and revised manuscript; R.S., A.A., D.E.A., and G.C.D.

505 approved final version of manuscript.

506

507 Competing interests: The authors declare no competing interests. 


\section{REFERENCES}

1. Andersen, R.A., Essick, G.K. \& Siegel, R.M. Encoding of spatial location by posterior parietal neurons. Science 230, 456-458 (1985).

$513 \quad 2 . \quad$ Avillac, M., Deneve, S., Olivier, E., Pouget, A. \& Duhamel, J.R. Reference frames for

514 representing visual and tactile locations in parietal cortex. Nat Neurosci 8, 941-949 (2005).

$515 \quad 3 . \quad$ Batista, A.P., Buneo, C.A., Snyder, L.H. \& Andersen, R.A. Reach plans in eye-centered 516 coordinates. Science 285, 257-260 (1999).

517 4. Duhamel, J.R., Bremmer, F., Ben Hamed, S. \& Graf, W. Spatial invariance of visual

518 receptive fields in parietal cortex neurons. Nature 389, 845-848 (1997).

519 5. $\quad$ Fetsch, C.R., Wang, S., Gu, Y., Deangelis, G.C. \& Angelaki, D.E. Spatial reference

520 frames of visual, vestibular, and multimodal heading signals in the dorsal subdivision of the medial superior temporal area. J Neurosci 27, 700-712 (2007).

6. Galletti, C., Battaglini, P.P. \& Fattori, P. Parietal neurons encoding spatial locations in craniotopic coordinates. Exp Brain Res 96, 221-229 (1993).

7. Jay, M.F. \& Sparks, D.L. Auditory receptive fields in primate superior colliculus shift with changes in eye position. Nature 309, 345-347 (1984). 8. Lee, J. \& Groh, J.M. Auditory signals evolve from hybrid- to eye-centered coordinates in the primate superior colliculus. $J$ Neurophysiol 108, 227-242 (2012).

9. Mullette-Gillman, O.A., Cohen, Y.E. \& Groh, J.M. Eye-centered, head-centered, and complex coding of visual and auditory targets in the intraparietal sulcus. J Neurophysiol 94, 530 2331-2352 (2005). 10. Mullette-Gillman, O.A., Cohen, Y.E. \& Groh, J.M. Motor-related signals in the intraparietal cortex encode locations in a hybrid, rather than eye-centered reference frame. Cereb Cortex 19, 1761-1775 (2009).

534 11. Schlack, A., Sterbing-D'Angelo, S.J., Hartung, K., Hoffmann, K.P. \& Bremmer, F. Multisensory space representations in the macaque ventral intraparietal area. $J$ Neurosci $\mathbf{2 5}$, 4616-4625 (2005). referenced representations of visual space in parietal cortex. Nature 394, 887-891 (1998). 13. Sajad, A., et al. Visual-Motor Transformations Within Frontal Eye Fields During HeadUnrestrained Gaze Shifts in the Monkey. Cereb Cortex 25, 3932-3952 (2015). 14. Kiesel, A., et al. Control and interference in task switching--a review. Psychol Bull 136, 849-874 (2010).

15. Ruge, H., Jamadar, S., Zimmermann, U. \& Karayanidis, F. The many faces of preparatory control in task switching: reviewing a decade of fMRI research. Hum Brain Mapp 34, 12-35 (2013).

16. Stoet, G. \& Snyder, L.H. Neural correlates of executive control functions in the monkey. Trends Cogn Sci 13, 228-234 (2009).

17. Stoet, G. \& Snyder, L.H. Single neurons in posterior parietal cortex of monkeys encode cognitive set. Neuron 42, 1003-1012 (2004). irrelevant variables by population decoding based on multisensory neurons. J Neurophysiol 116, 1449-1467 (2016). 
19. Sasaki, R., Angelaki, D.E. \& DeAngelis, G.C. Dissociation of Self-Motion and Object Motion by Linear Population Decoding That Approximates Marginalization. J Neurosci 37, 11204-11219 (2017). 20. Rushton, S.K. \& Warren, P.A. Moving observers, relative retinal motion and the detection of object movement. Curr Biol 15, R542-543 (2005).

21. Warren, P.A. \& Rushton, S.K. Optic flow processing for the assessment of object movement during ego movement. Curr Biol 19, 1555-1560 (2009).

22. Royden, C.S. \& Connors, E.M. The detection of moving objects by moving observers. Vision Res 50, 1014-1024 (2010).

23. Royden, C.S. \& Holloway, M.A. Detecting moving objects in an optic flow field using direction- and speed-tuned operators. Vision Res 98, 14-25 (2014).

24. Fajen, B.R. \& Matthis, J.S. Visual and non-visual contributions to the perception of object motion during self-motion. PLoS One 8, e55446 (2013).

25. Dokka, K., MacNeilage, P.R., DeAngelis, G.C. \& Angelaki, D.E. Multisensory selfmotion compensation during object trajectory judgments. Cereb Cortex 25, 619-630 (2015).

26. MacNeilage, P.R., Zhang, Z., DeAngelis, G.C. \& Angelaki, D.E. Vestibular facilitation of optic flow parsing. PLoS One 7, e40264 (2012).

27. Eifuku, S. \& Wurtz, R.H. Response to motion in extrastriate area MSTl: center-surround interactions. J Neurophysiol 80, 282-296 (1998).

28. Tanaka, K., Sugita, Y., Moriya, M. \& Saito, H. Analysis of object motion in the ventral part of the medial superior temporal area of the macaque visual cortex. J Neurophysiol 69, 128142 (1993).

29. Ilg, U.J., Schumann, S. \& Thier, P. Posterior parietal cortex neurons encode target motion in world-centered coordinates. Neuron 43, 145-151 (2004).

30. Chen, X., DeAngelis, G.C. \& Angelaki, D.E. Diverse spatial reference frames of vestibular signals in parietal cortex. Neuron 80, 1310-1321 (2013).

31. Chen, X., DeAngelis, G.C. \& Angelaki, D.E. Eye-centered representation of optic flow tuning in the ventral intraparietal area. J Neurosci 33, 18574-18582 (2013).

32. Berens, P., et al. A fast and simple population code for orientation in primate V1. $J$ Neurosci 32, 10618-10626 (2012).

33. Zaidel, A., DeAngelis, G.C. \& Angelaki, D.E. Decoupled choice-driven and stimulusrelated activity in parietal neurons may be misrepresented by choice probabilities. Nat Commun 8, 715 (2017).

34. Britten, K.H., Newsome, W.T., Shadlen, M.N., Celebrini, S. \& Movshon, J.A. A relationship between behavioral choice and the visual responses of neurons in macaque MT. Vis Neurosci 13, 87-100 (1996).

35. Dokka, K., DeAngelis, G.C. \& Angelaki, D.E. Multisensory Integration of Visual and Vestibular Signals Improves Heading Discrimination in the Presence of a Moving Object. $J$ Neurosci 35, 13599-13607 (2015).

36. Sasaki, R., Angelaki, D.E. \& DeAngelis, G.C. Processing of object motion and selfmotion in the lateral subdivision of the medial superior temporal area in macaques. $J$ Neurophysiol 121, 1207-1221 (2019).

37. Chen, A., DeAngelis, G.C. \& Angelaki, D.E. Functional specializations of the ventral intraparietal area for multisensory heading discrimination. J Neurosci 33, 3567-3581 (2013). 
597 38. Gu, Y., et al. Perceptual learning reduces interneuronal correlations in macaque visual 598 cortex. Neuron 71, 750-761 (2011).

599 39. Kohn, A., Coen-Cagli, R., Kanitscheider, I. \& Pouget, A. Correlations and Neuronal

600 Population Information. Annu Rev Neurosci 39, 237-256 (2016).

601 40. Averbeck, B.B., Latham, P.E. \& Pouget, A. Neural correlations, population coding and 602 computation. Nat Rev Neurosci 7, 358-366 (2006).

603 41. Moreno-Bote, R., et al. Information-limiting correlations. Nat Neurosci 17, 1410-1417 604 (2014).

605 42. Dokka, K., Park, H., Jansen, M., DeAngelis, G.C. \& Angelaki, D.E. Causal inference

606 accounts for heading perception in the presence of object motion. Proc Natl Acad Sci U S A 116, 607 9060-9065 (2019).

608 43. Chen, X., DeAngelis, G.C. \& Angelaki, D.E. Eye-centered visual receptive fields in the 609 ventral intraparietal area. $J$ Neurophysiol 112, 353-361 (2014).

610 44. Chen, X., DeAngelis, G.C. \& Angelaki, D.E. Flexible egocentric and allocentric

611 representations of heading signals in parietal cortex. Proc Natl Acad Sci US A 115, E3305612 E3312 (2018).

613 45. Crespi, S., et al. Spatiotopic coding of BOLD signal in human visual cortex depends on 614 spatial attention. PLoS One 6, e21661 (2011).

615 46. Merriam, E.P., Gardner, J.L., Movshon, J.A. \& Heeger, D.J. Modulation of visual 616 responses by gaze direction in human visual cortex. J Neurosci 33, 9879-9889 (2013).

617 47. Bernier, P.M. \& Grafton, S.T. Human posterior parietal cortex flexibly determines 618 reference frames for reaching based on sensory context. Neuron 68, 776-788 (2010).

619 48. Bremner, L.R. \& Andersen, R.A. Temporal analysis of reference frames in parietal cortex 620 area 5d during reach planning. J Neurosci 34, 5273-5284 (2014).

621 49. Duncker, K. Uber induzierte Bewegung. Psychologische Forschung 12, 180-259 (1929).

622 50. Zivotofsky, A.Z. The Duncker illusion: intersubject variability, brief exposure, and the 623 role of eye movements in its generation. Invest Ophthalmol Vis Sci 45, 2867-2872 (2004). 


\section{FIGURE LEGENDS}

626 Figure 1. Schematic illustration of interactions between object motion and self-motion. (a)

627 An object (gray sphere) moves upward in the world while an observer is translated rightward or

628 leftward at two speeds. (b) Resultant image motion vectors. Without self-motion, image motion

629 is upward (white). During self-motion, image motion is biased according to the direction and

630 speed of self-motion. For simplicity, the image view in panel $b$ does not reflect image reversals

631 that would be caused by projection onto the retina.

633 Figure 2. Behavioral task design and predicted psychometric functions. (a) A sphere of dots

634 moves up-right $(+\theta)$ or up-left $(-\theta)$ in the world. Rightward or leftward self-motion occurs while

635 the animal views the moving object. (b) In the world coordinate task, a world-fixed partial cube

636 indicates that the monkey should report object motion relative to the world. In the head

637 coordinate task, the partial cube remains fixed relative to the head, and cues a report in head

638 coordinates. Dashed vertical lines indicate fixed world-centered locations as a reference.

639 Background dots were presented at 40\% coherence, but background motion here is depicted with

$640100 \%$ coherence for visual clarity. (c) Time course of the luminance of visual stimulus

641 components. The luminance of the object and partial cube were changed dynamically such that

642 the partial cube faded out during the portion of the trial when the object faded in. (d)

643 Hypothetical psychometric functions that plot the proportion of 'rightward' choices as a function

644 of object direction in world coordinates. If the animal compensates fully for self-motion in the

645 world coordinate task, psychometric functions for rightward and leftward self-motion should

646 overlap (magenta). On the other hand, those functions should shift with self-motion by a specific 
647 amount (horizontal bar) in the head coordinate task (cyan). Dashed/solid curves:

648 leftward/rightward self-motion.

650 Figure 3. Summary of behavioral performance for each task reference frame. (a)

651 Psychometric functions showing the proportion of rightward choices as a function of object

652 direction in world coordinates (positive = rightward). Data are shown for trials in which there is

653 no self-motion (Object Only, black), for trials with self-motion in which the animal performs the

654 head coordinate task (cyan/navy), and for trials in which the animal performs the world-

655 coordinate task (magenta/brown). Darker colors (navy/brown) represent the Object+Visual

656 condition and lighter colors (cyan/magenta) represent the Object+Combined condition.

657 Filled/open symbols: rightward/leftward self-motion. Smooth curves are cumulative Gaussian

658 fits to data pooled across 128 sessions for Monkey $\mathrm{N}$ and 57 sessions for monkey K. (b)

659 Summary of behavioral biases, quantified as the difference in point of subjective equality

660 ( $\triangle \mathrm{PSE}$ ) between psychometric functions for rightward and leftward self-motion. $\triangle \mathrm{PSE}$ values

661 are compared between the Object+Combined and Object + Visual conditions for each recording

662 session and each monkey (squares: monkey N; diamonds: monkey K). Data are shown separately

663 for the world coordinate (red) and head coordinate (blue) task conditions. Error bars represent

$66495 \%$ confidence intervals around the mean values (black symbols) across 185 sessions.

665

666 Figure 4. Data from example neurons recorded from areas VIP and MSTl. (a) Data from

667 two example VIP neurons (one neuron per column) recorded in the Object+Combined condition.

668 The top and bottom rows plot firing rates as a function of object direction in head and world

669 coordinates, respectively. (b) Data from two example MSTl neurons in the Object+Combined 
670 condition. Note the greater differences in response between the head (cyan) and world (magenta)

671 coordinate task conditions for the example VIP neurons, as compared to the example MST1

672 neurons. Error bars denote SEM (across n=10 stimulus repetitions). (c, d) Data for the same 4

673 example neurons from the Object+Visual condition.

675 Figure 5. Summary of single-unit results for VIP and MSTl. (a) Summary of modulation

676 index (MI) values for populations of neurons recorded from VIP (orange, N=223) and MST1

677 (green, $\mathrm{N}=177$ ) in the Object+Visual (top) and Object+Combined (bottom) conditions. MI

678 measures the response difference between a pair of object tuning curves in the head- and world-

679 coordinate tasks. Filled bars represent MI values significantly greater than zero (permutation test,

$680 \mathrm{p}<0.05)$. Numbers in the legend indicate the total number of neurons, as well as the number

681 with MI values significantly greater than zero, for each brain area. Arrowheads and numbers

682 indicate the median values for each brain area and self-motion condition. (b) Time course of

683 average MI values for VIP (orange, N=223) and MSTl (green, N=177) neurons in the

684 Object+Visual (lighter hues) and Object+Combined (darker hues) conditions. Error bars

685 represent $95 \%$ confidence intervals. Gray curve shows the Gaussian temporal profile of object

686 speed. (c) The correlation between object direction tuning (computed in world coordinates) is

687 compared for the world and head coordinate tasks. Data from VIP and MSTl are shown in

688 orange and green, respectively. Data are included in this panel only for neurons (VIP: N=57;

689 MSTl: $\mathrm{N}=44$ ) that had significant tuning (ANOVA, $\mathrm{p}<0.05$ ) in the Object Only condition. Star

690 symbols denote the three neurons in Figure 4 that met this criterion. (d) Time course of the

691 difference in tuning correlation between world and head coordinate tasks for the same 
692 populations of VIP (orange, $\mathrm{N}=223$ ) and MSTl (green, $\mathrm{N}=177$ ) neurons described in panel c.

693 Error bars represent 95\% confidence intervals.

694

695 Figure 6. Summary of population decoding results. Panels a-c correspond to results from

696 training separate decoders to perform the world and head coordinate tasks; panels d-f correspond

697 to results from a single decoder trained to perform in both task reference frames. (a) Schematic

698 diagram of separate decoders for the head and world coordinate task conditions. (b) Results for

699 separate world/head task decoders, plotted in the same format as the behavioral data of Fig. 3a.

700 Decoding VIP activity produces a pattern of results very similar to behavior, whereas decoding

701 MSTl produces large biases in the world coordinate task. (c) Summary comparison of monkey

702 behavior and performance of the separate decoders. $\triangle \mathrm{PSE}$ for the Object+Combined condition is

703 plotted against $\triangle \mathrm{PSE}$ for the Object+Visual condition. Results from the VIP decoder (orange) are

704 largely similar to behavior (black, same data from Fig. 3b), whereas results from the MST1

705 decoder (green) depart sharply from behavioral performance for the world coordinate task. Error

706 bars on decoder performance values represent $95 \%$ confidence intervals obtained by

707 bootstrapping ( $\mathrm{n}=1000$ bootstraps, see Methods). Pink and cyan dashed lines: expected $\Delta$ PSE for

708 perfect performance in the world and head coordinate tasks, respectively. (d) Schematic diagram

709 for the single decoder. (e) Analogous results to panel b, but from a single decoder trained to

710 classify object direction in both task reference frames. (f) Summary comparison of single

711 decoder results with behavior. Format as in panel c; error bars represent $95 \%$ confidence

712 intervals across $n=1000$ bootstraps. 
714 Figure 7. Time course of decoder performance. (a) Schematic illustration of examples of

715 "response matched" and "response conflict" conditions. In a response matched condition (left),

716 correct answers are the same for both task reference frames; in a response conflict condition

717 (right), correct reports are opposite for the two reference frames. Magenta and cyan vectors

718 indicate object direction in world and head coordinates, respectively. (b) Time course of decoder

719 classification accuracy for populations of VIP (left, n=223) and MSTl (right, n=177) neurons,

720 evaluated in the subset of response conflict conditions. Separate decoders were trained to classify

721 object direction in the world (magenta/brown) and head (cyan/navy) reference frames at each

722 time point. Error bars represent 95\% confidence intervals (across $n=100$ bootstraps). (c) Time

723 course of decoder classification accuracy in the subset of response matched conditions, format as

724 in b. Time courses were obtained by computing each variable within a $300 \mathrm{~ms}$ sliding time

725 window that was advanced across the trial epoch in steps of $50 \mathrm{~ms}$.

727 Figure 8. Time courses of classification accuracy using within-task vs. cross-task decoding.

728 (a, b) Results for decoding VIP activity. In panel a, the decoder is trained to classify object

729 direction in world coordinates using responses from the world task condition (magenta/brown,

730 within-task) or using responses from the head task condition (light/dark gray, cross-task). In

731 panel $b$, the decoder is trained to classify object direction in head coordinates using responses

732 from the head task condition (cyan/navy, within-task) or the world task condition (light/dark gray,

733 cross-task). (c,d) Analogous results for within-task (colors) and cross-task (gray) decoders of

734 MSTl activity. In all panels, error bars denote 95\% confidence intervals (across $n=100$

735 bootstraps). 


\section{METHODS}

\section{General}

740 Two male rhesus monkeys (Macaca mulatta) participated in this study. During this study, 741 monkey $\mathrm{K}$ ranged in age from 4 to 6 years and ranged in weight from 5.8 to $8.5 \mathrm{~kg}$. Monkey $\mathrm{N}$

742 ranged in age from 5 to 7 years and weight from 7.2 to $9.7 \mathrm{~kg}$. General procedures have been

743 described previously ${ }^{19,51}$. All experimental procedures conformed to National Institutes of

744 Health guidelines and were approved by the University Committee on Animal Resources at the

745 University of Rochester. Additional information can be found in the Life Sciences Reporting

746 Summary.

747

$748 \quad$ Vestibular and visual stimuli

749 A 6 degree-of-freedom motion platform (MOOG 6DOF2000E; Moog) was used to passively

750 translate animals leftward or rightward along the interaural axis. Visual stimuli were projected

751 onto a tangent screen by a three-chip digital light projector (Mirage $\mathrm{S}^{+} 3 \mathrm{~K}$; Christie Digital

752 Systems, Cypress, CA). The display screen measured $60 \times 60 \mathrm{~cm}$ and was mounted $\sim 30 \mathrm{~cm}$ in

753 front of the monkey, thus subtending $\sim 90 \times 90^{\circ}$ of visual angle. Visual stimuli simulated

754 translational self-motion through a three-dimensional field of stars. Each star was a triangle that

755 measured $0.15 \mathrm{~cm} \times 0.15 \mathrm{~cm}$, and the field of stars measured $100 \mathrm{~cm}$ wide by $100 \mathrm{~cm}$ tall by 40

$756 \mathrm{~cm}$ deep, with a star density of 0.01 stars per $\mathrm{cm}^{3}$. To provide stereoscopic cues, the star field

757 was rendered as a red-green anaglyph and viewed through custom red-green goggles, consisting

758 of Kodak Wratten2 filters (\#29 and \#61). The entire display was visible through the colored

759 filters. 
The optic flow field contained naturalistic cues simulating lateral translation of the observer

761 in the horizontal plane; these included motion parallax, size, and binocular disparity cues. While

762 the monkey was translated leftward or rightward, an object also moved upward in the world with

763 a small leftward or rightward component (Fig. 1). The moving object was a transparent sphere

764 (diameter $\left.10^{\circ}\right)$ composed of random dots, with a density $\left(0.25 \mathrm{dots} / \mathrm{cm}^{3}\right)$ that was higher than

765 that of the star-field background, such that the object was easily segmented from the background.

766 The moving object's center was located in depth within the plane of the visual display, such that

767 it consisted of dots with a mixture of crossed and uncrossed disparities. At the start of each trial,

768 the object appeared with its center located 5 deg left of fixation and 10 deg below fixation. The

769 object moved in one of 7 directions relative to upward in the world: $-21,-14,-7,0,7,14$ and 21

$770 \mathrm{deg}$, where negative angles represent upward/left motion, positive angles represent upward/right

771 motion, and 0 means straight upward (in world coordinates). All self-motion and object motion

772 trajectories were straight translational movements with a duration of $2 \mathrm{sec}$, and having a

773 Gaussian velocity profile with a SD of $1 / 3 \mathrm{sec}^{51}$. Peak stimulus velocity occurred at $1120 \mathrm{~ms}$

774 after stimulus onset, due to delays and dynamics of the motion platform; visual stimuli were

775 synchronized to platform motion. The total excursion $(0.25 \mathrm{~m})$ and peak velocity $(0.75 \mathrm{~m} / \mathrm{s})$ of

776 object motion were greater than those for self-motion $(0.08 \mathrm{~m}$ and $0.24 \mathrm{~m} / \mathrm{s}$, respectively).

777 Because the head-centered velocity of the object is determined by both self-motion velocity and

778 object velocity relative to the world, the object could move up/left in world coordinates and

779 up/right in head coordinates, or vice-versa. While we shall distinguish between world- and head-

780 centered references frames in this study, we cannot distinguish head-centered and retinal

781 reference frames because the fixation target was always head-fixed. 
Two different versions of the task were interleaved that required the animal to report object

783 direction in either head or world coordinates (Fig. 2). In the world coordinate task (Fig. 2b), a

784 partially-visible cube defined a world-fixed reference frame that was updated every video frame.

785 The cube dimensions were $76 \mathrm{~cm}$ wide by $76 \mathrm{~cm}$ tall by $40 \mathrm{~cm}$ deep and the center of the cube

786 was located in depth at the fixation point. Thus, the cube moved relative to the head during self-

787 motion in the world coordinate task (Fig. 2b) but remained head-fixed in the head coordinate task

788 (Fig. 2b).

789 We attempted to train monkeys to switch between the world and head coordinate tasks

790 based solely on the color of the fixation point. While one animal could partially achieve this, the

791 other animal could not. Both animals were much better able to switch between tasks when the

792 partially-visible cube was presented. We think that the partial cube was particularly important

793 because it was not possible to eliminate luminance boundaries at the edge of the display. Since

794 the display screen translated with the animal, the luminance boundaries always provided a head-

795 centered reference frame, and thus the partially visible cube was important to help define a world

796 reference frame (anecdotally, this was the case for human observers also).

797 A potential concern about use of the partially visible cube is that animals might learn to

798 report object direction relative to the moving visual elements of the cube. Two measures helped

799 to prevent this possibility. First, the luminance of the moving object was dynamically changed

800 according to the same Gaussian envelope that governed the speed of object and self-motion, such

801 that the moving object was initially invisible, reached maximum brightness in the middle of the

802 presentation when it also reached maximum speed, and then decayed again to become invisible

803 at the end of the trial (Fig. 2b,c). Simultaneously, the luminance of the partially visible cube

804 followed an inverted Gaussian velocity profile, such that the cube was maximally visible at the 
805 beginning and end of each trial and disappeared in the middle of the trial (Fig. 2b,c). This

806 allowed the partial cube to define the reference frame while having little overlap with the

807 visibility of the moving object.

808 Second, to assess whether animals might have still judged object motion relative to the 809 partially-visible cube, we performed behavioral control experiments in which we randomly

810 varied the location of the partial cube in depth from trial to trial. If animals reported object

811 direction relative to the cube, then their performance should depend systematically on the depth

812 of the cube. We found no such dependence (Extended Data Fig. 10), indicating that animals

813 were successfully prevented from adopting this strategy. Thus, we believe that both animals

814 successfully learned to report object direction in world or head coordinates.

\section{Behavioral task}

817 Monkeys were trained to report whether the object moved up-left or up-right in either head-

818 or world-coordinates (Fig. 2). This was a very challenging task for animals to learn, and required

$819 \quad 1.5-2$ years of training for each animal. We initially trained the animals to perform the head and

820 world tasks in separate blocks of trials. We then gradually reduced the length of these blocks,

821 and then transitioned animals to trial-by-trial interleaving of the two tasks.

822 In each trial, a fixation point initially appeared. Once the monkey looked at the fixation

823 point, the object appeared and moved upward in the virtual environment, with a small rightward

824 or leftward component. The monkey reported whether the object moved upward/rightward or

825 upward/leftward by making a saccade to one of two targets that appeared (10 degrees to the right

826 and left of the fixation target) after a $500 \mathrm{~ms}$ delay period following the end of the visual

827 stimulus. In the world and head coordinate tasks (Fig. 2b), the monkey reported whether object 
828 motion moved leftward or rightward relative to vertical in world or head coordinates,

829 respectively. The two versions of the task were cued by the shape and color of the fixation point

830 (Fig. 2b), such that they could be randomly interleaved. Animals were rewarded for reporting

831 the correct direction of object motion in each reference frame condition. When object direction

832 was exactly vertical (in the relevant reference frame), monkeys were rewarded randomly on $50 \%$

833 of trials.

834 Crucially, for each particular combination of a self-motion direction and an object motion

835 direction in the world, the motion trajectory of the object in head (or retinal) coordinates was

836 identical across the two task conditions. Thus, for the same exact motion of all of the dots on the

837 screen, the animal might be required to make a rightward choice in the world coordinate task and

838 a leftward choice in the head coordinate task, or vice-versa. For other stimulus conditions, the

839 correct choice would be the same in both reference frames. This allowed us to compare decoder

840 performance for subsets of trials in which the correct answers were the same or different for the

841 two tasks.

842 Three self-motion conditions were interleaved for each task reference frame. 1) In the Object

843 Only condition, there was no self-motion such that world- and head-centered reference frames

844 are aligned. Background dots were stationary on the display in the Object Only condition, since

845 the only source of image motion for the background dots is self-motion. 2) In the Object+Visual

846 condition, the motion platform remained stationary while a background of random dots provided

847 optic flow that simulated leftward or rightward self-motion. Background optic flow had a motion

848 coherence of $40 \%$ such that the object was easy to segment from the background. 3 ) In the

849 Object+Combined condition, self-motion was indicated by both optic flow and physical

850 translation of the motion platform (which provided vestibular cues). Since cue combination is 
851 known to enhance heading perception ${ }^{52,53}$, we expected that the monkeys would be best able to

852 compensate for self-motion in this condition.

853

854 Behavioral data analysis

855 Psychometric functions were constructed by plotting the proportion of 'rightward' choices 856 as a function of object direction in world coordinates. Plotted in this fashion, psychometric

857 functions for rightward and leftward self-motion should overlap in the world coordinate task if 858 the animal compensates fully for self-motion (Fig. 2d). If the animal does not account for self-

859 motion and reports object direction in head coordinates, there will be a large horizontal shift

860 between psychometric functions corresponding to leftward and rightward self-motion (Fig. 2d).

861 To quantify these shifts, we fit each psychometric curve with a cumulative Gaussian function

862 and used the mean parameter of the fit to estimate the point of subjective equality (PSE) for each

863 direction of self-motion. The difference in PSE ( $\triangle \mathrm{PSE}$ ) between rightward and leftward self-

864 motion directions was then taken as an index of the reference frame used by the monkeys to

865 judge object direction (Fig. 3b). If the monkey correctly estimates object direction in head

866 coordinates, then we expect $\triangle \mathrm{PSE}=35.5 \mathrm{deg}$ (horizontal bar, Figs. 2d, 3a).

\section{Physiological recording procedures}

869 Neural recordings were obtained from the right hemisphere of two monkeys while the

870 animals performed the behavioral task. We recorded 223 VIP neurons (monkey N, N=93;

871 monkey K, N=130) and 177 MSTl neurons (monkey N, N=94; monkey K, N=83), with most

872 neurons recorded in separate sessions. We attempted to record from any VIP and MSTl neuron

873 that could be isolated; there were no selection criteria based on response properties other than 
874 receptive field location. Recordings were included if we obtained data for at least 3 repetitions

875 for each stimulus condition. For Monkey N, 68 VIP and 50 MSTl neurons were recorded with

876 single tungsten microelectrodes (FHC, Bowdoinham, ME; 0.5 - $1 \mathrm{M} \Omega$ impedance). Single-unit

877 action potentials were sorted on-line using a hardware window discriminator (Bak Electronics).

878 The remaining 25 VIP and 44 MSTl neurons from Monkey N were recorded with linear

879 electrode arrays that were inserted into either VIP or MST1 daily (Plexon U-probes with two

880 rows of 12 channels spaced $100 \mu \mathrm{m}$ vertically and $50 \mu \mathrm{m}$ horizontally or Plexon V-probes with

88124 channels spaced $50 \mu \mathrm{m}$ vertically). For array recordings, single-unit action potentials were

882 isolated using Plexon Offline Sorter. For Monkey K, all 130 VIP and 83 MSTl neurons were

883 recorded with linear arrays. There were a total of 104 recording sessions for VIP (monkey N,

$884 \mathrm{~N}=69$; monkey $\mathrm{K}, \mathrm{N}=35$ ) and 81 recording sessions for MSTl (monkey $\mathrm{N}, \mathrm{N}=59$; monkey $\mathrm{K}$,

$885 \mathrm{~N}=22$ ). In experiments using linear arrays, a mean of 3.8 and 3.7 neurons were recorded

886 simultaneously for areas MSTl and VIP, respectively.

887 Both VIP and MSTl were initially localized via structural MRI scans as described previously

888 for VIP ${ }^{54}$ and MST ${ }^{55}$. We were careful to distinguish MSTl from the dorsal subdivision of area

889 MST (MSTd) and MT ${ }^{36}$. To do this, we carefully mapped the portions of area MT that were

890 found beneath MSTd, in the posterior bank of the superior temporal sulcus. We located the

891 foveal representation of area MT, which is generally located at the anterior-lateral extent of MT.

892 We then carefully mapped regions around that area, and MSTl was localized primarily in regions

893 anterior to the foveal representation of MT.

894

895 Experimental protocol 
896 We first performed standard tests to map receptive fields and assess response properties

897 qualitatively. These tests, along with mapping recording sites onto structural MRI images ${ }^{54}$,

898 allowed us to confidently assign recording sites to MST1 or VIP. Neurons were isolated while

899 presenting a large field of flickering dots that could be varied in position, size, and velocity. For

900 some neurons, we used a reverse-correlation technique to measure the spatial and directional

901 receptive field structure of each neuron ${ }^{56}$. From these maps, we fit the receptive field with a

902 two-dimensional Gaussian, and used the contour of the Gaussian at half-maximal response to

903 define the receptive field contours shown in Extended Data Fig. 2 (17\% of VIP neurons and 13\%

904 of MSTl neurons). Due to a technical difficulty, reverse correlation maps were not available for a

905 substantial fraction of neurons. In other recordings, receptive fields were mapped by hand, and

906 receptive field location and size was estimated when the map was clearly noted. We also

907 performed a standard measurement of directional tuning within the fronto-parallel plane by

908 presenting 8 directions of motion, 45 deg apart. These preliminary tests typically required 150-

909200 trials of fixation behavior.

910 We recorded from all neurons regardless of their direction and speed preferences. To

911 facilitate population decoding, we used exactly the same stimulus set for all recorded neurons.

912 This allowed us to construct pseudo-population responses for decoding, although these pseudo-

913 population responses do not contain accurate correlated noise since the vast majority of neurons

914 were recorded separately. After extensively mapping out the receptive field coverage of neurons

915 in areas VIP and MSTl, we focused our recordings on a set of penetrations for which receptive

916 fields were concentrated on the same region of space for both brain areas (Extended Data Fig. 2).

917 We carefully selected the starting location and trajectory of object motion based on the

918 distributions of receptive fields of MSTl and VIP neurons in our selected penetrations, such that 
919 object motion was centered on the receptive field locations for the populations of neurons in both

920 VIP and MSTl (Extended Data Fig. 2). The main experimental protocol involved 7 directions of

921 object motion, 2 reference frame conditions (head or world), 2 self-motion directions, and 10

922 stimulus repetitions for each of the Object+Visual and Object+Combined conditions (560 trials),

923 as well as 7 directions and 20 stimulus repetitions for the Object Only condition (140 trials), for a

924 total of 700 trials.

925

926 Neural data analyses

927 Neural responses were computed as firing rates over a time window from 500-2500 ms

928 following stimulus onset. Since the stimulus duration was $2000 \mathrm{~ms}$, this window included most

929 of the stimulus period during which neurons were active, as well as the 500ms delay period after

930 stimulus offset. This analysis window was based on inspection of population response profiles

931 (Extended Data Fig. 4a). The initial 500ms of the stimulus period was not included in our main

932 analysis window because there is an early response to luminance onset of dots during this time

933 (see Extended Data Fig. 4a) and because object and background motion is small during the first

$934500 \mathrm{~ms}$ (due to the Gaussian velocity profile used). Our main analyses were also conducted as a

935 function of time, using a moving window of $300 \mathrm{~ms}$ that was slid across the data in steps of 50

936 ms. All analyses are performed on all trials, including both correct and incorrect trials, unless

937 indicated otherwise.

938 Modulation Index for the effect of reference frame on neural responses: To quantify how

939 neural responses are modulated by the task reference frame, we computed a modulation index

940 (MI) as follows: 


$$
M I=\frac{1}{N}\left(\frac{\left|\sum_{\theta}\left(R(\theta)_{W, L}-R(\theta)_{H, L}\right)\right|+\left|\sum_{\theta}\left(R(\theta)_{W, R}-R(\theta)_{H, R}\right)\right|}{\left|\sum_{\theta}\left(R(\theta)_{W, L}+R(\theta)_{H, L}\right)\right|+\mid \sum_{\theta}\left(R(\theta)_{W, R}+R(\theta)_{H, R}\right)}\right)
$$

942 In this formulation, $\mathrm{R}_{\mathrm{W}}$ and $\mathrm{R}_{\mathrm{H}}$, denote the mean responses of a neuron in the world and head-

943 coordinate tasks, respectively, whereas additional subscripts L and R denote leftward and

944 rightward self-motion directions. $\theta$ represents object direction, and $\mathrm{N}$ denotes the number of

945 object directions. MI ranges from 0 (no difference between responses in the two reference

946 frames) to 1 (if, for example, responses to one reference frame condition are completely

947 suppressed).

948 In formulating MI, we sought a simple metric to quantify response modulations related to

949 the task reference frame across all object and self-motion directions. If neural responses were

950 identical in the world and head coordinate tasks, MI would be zero; however, in practice, MI

951 values are unlikely to be very close to zero due to response variability. If world and head

952 coordinate tasks produce different average neural responses, then MI values will become

953 substantially greater than zero. Note that MI is not sensitive to the nature of response

954 modulations (e.g., peak shifts vs. gain modulations vs. tuning shape changes). Given that our

955 direction discrimination task covered a relatively narrow range of directions relative to the full

956 tuning curves, it is difficult to examine the exact nature of tuning changes from our data.

957 Direction discrimination index: To quantify the strength of tuning for object direction, we

958 used a direction discrimination index (DDI) that was defined as follows:

$$
D D I=\frac{R_{\text {max }}-R_{\text {min }}}{R_{\text {max }}-R_{\text {min }}+2 \sqrt{S S E /(N-M)}}
$$

960 where $R_{\max }$ and $R_{\min }$ represent the maximum and minimum responses from the measured

961 direction tuning function, respectively. $S S E$ is the sum squared error around the mean responses, 
$962 N$ is the total number of observations (trials), and $M$ is the number of tested object directions ( $M$

$963=7)$. DDI is a signal-to-noise metric, conceptually similar to d', that is normalized to range from

9640 to 1 . Neurons with stronger response modulations relative to their variability will take on

965 values closer to 1.

966 Effect of partial cube frame on responses: To quantify effects of the partial cube frame on

967 neural responses, we computed a cube effect index (CEI). This index measures neural responses

968 over the initial 500ms of each trial, when the partial cube is visible while background dots are

969 largely invisible (Fig. 2c). For each object direction, $\theta$, CEI takes the absolute difference in

970 response to the cube frame between rightward and leftward self-motion directions, and divides

971 by the sum of those responses. The resultant is then averaged across the $N$ object directions. For

972 the world coordinate task, the calculation of CEI is as follows:

973

$$
C E I_{W}=\frac{1}{N}\left(\frac{\left|\Sigma_{\theta}\left(R(\theta)_{W, R}-R(\theta)_{W, L}\right)\right|}{\left|\sum_{\theta}\left(R(\theta)_{W, R}+R(\theta)_{W, L}\right)\right|}\right)_{t \in[0-500 m s]}
$$

974 where $R_{W}$ denotes the mean responses of a neuron in the world task, and subscripts $L$ and $R$

975 denote leftward and rightward self-motion directions. The calculation of CEI for the head

976 coordinate task is identical, with replacing $R_{W}$ by $R_{H}$.

977 Metrics of choice-related and task-related activity: To quantify choice-related activity in

978 single neurons, we computed the well-established choice probability (CP) metric ${ }^{34}$. For each

979 unique object direction, self-motion direction, self-motion modality (visual, combined), and task

980 reference frame condition (world vs. head), the distribution of responses was z-scored and then

981 divided into two groups based on whether the animal made a leftward or rightward saccade. Z-

982 scored responses were then pooled across unique stimulus/task conditions as long as there were

983 at least 3 choices made in each direction. ROC analysis was then applied to the pooled z-scores 
984 for the two choice groups, and CP was defined as the area under the ROC curve. For our

985 purposes, CP was not referenced to each neuron's preferred direction; rather $\mathrm{CP}>0.5$

986 corresponds to a preference for rightward choices and $\mathrm{CP}<0.5$ corresponds to a preference for

987 leftward choices. This avoids potential issues with defining the "preferred" stimulus when choice

988 effects are large ${ }^{33}$.

989 We devised an analogous ROC-based metric to quantify single-unit activity related to task

990 reference frame. This 'task probability' (TP) metric is computed just like CP, but swapping the

991 roles of variables that represent choice (left vs. right) and task (head vs. world). For each distinct

992 combination of object direction, self-motion direction, self-motion modality, and choice,

993 responses were z-scored and sorted into two groups based on task reference frame. If there were

994 at least 3 trials for world and head reference frames, normalized responses from that condition

995 were pooled with other conditions that met the same criteria. ROC analysis was applied to the

996 pooled $\mathrm{z}$-scores that were sorted into world and head task groups. TP $>0.5$ corresponds to

997 greater responses in the world coordinate task, and TP $<0.5$ corresponds to greater responses in

998 the head coordinate task.

999 Removal of choice- and task-related response modulations: To test whether choice- or task-

1000 related signals make specific contributions to decoder performance, we devised a method to

1001 remove either choice- or task-related response modulations from neural activity. First, we

1002 identified a set of trials corresponding to each unique combination of object direction, self-

1003 motion direction, and self-motion modality. If this set of trials included at least 3 trials each for

1004 left and right choices and 3 trials each for world and head task conditions, then we proceeded to

1005 remove either the choice- or task-related response component. To remove the choice-related

1006 response component, we shifted the mean responses for right and left choices toward each other 
1007 to equate the mean responses. Comparison of Extended Data Fig. 9a,b to Extended Data Fig.

1008 8a,b indicates that this manipulation eliminated most of the choice-related modulations, while

1009 preserving task-related modulations. Similarly, to remove the task-related component, we shifted

1010 the mean responses for world and head task conditions to equate the means. Extended Data Fig.

$10119 \mathrm{c}, \mathrm{d}$ indicates that this manipulation was successful in eliminating most of the task-related

1012 modulations, while preserving choice effects. These manipulations cannot completely remove all

1013 choice- or task-related activity because they can only be performed when there are at least a few

1014 trials in each choice and task group, and estimates of mean responses based on a few trials are

1015 noisy.

1016 Population decoding by a linear classifier: Linear decoding was performed to classify object

1017 direction as rightward or leftward of vertical in each reference frame. Pseudo-population

1018 responses of 223 neurons for VIP and 177 neurons for MST1 were used for this purpose. We

1019 used a linear classifier to categorize object motion as rightward or leftward relative to vertical in

1020 either world or head coordinates:

$$
f=\sum_{i=1}^{N} w_{i} \cdot r_{i}+\mathrm{k}
$$

1022 Here, $N$ is the number of neurons in the pseudo-population for either VIP or MTl, $r_{i}$ is the

1023 response of the $i^{\text {th }}$ neuron, $w_{i}$ is the decoding weight for the $i^{\text {th }}$ neuron, and $k$ is constant scalar.

1024 The decoder's choice is determined by the sign of the output variable, $f$. We used a Fisher linear

1025 discriminant (FLD) to compute the parameters $(w, k)$ as follows:

1026

$$
\begin{gathered}
w=\Sigma^{-1} \cdot\left(\mu_{R}-\mu_{L}\right) \\
k=\frac{1}{2} \cdot\left[\left(\mu_{L}^{T} \cdot \Sigma^{-1} \cdot \mu_{L}\right)-\left(\mu_{R}^{T} \cdot \Sigma^{-1} \cdot \mu_{R}\right)\right]
\end{gathered}
$$


1028 where $\mu_{L}$ and $\mu_{R}$ indicate the mean population response vectors for rightward and leftward object

1029 directions relative to the world (for the world task decoder) or head (for the head task decoder),

1030 and $\Sigma$ is the response covariance matrix.

1031 Since most of our neurons were not recorded simultaneously, all neurons did not see the 1032 same number of repetitions of each unique stimulus. Thus, we constructed a population response 1033 matrix in which each neuron had responses corresponding to 10 stimulus repetitions. For neurons 1034 recorded for $>10$ repetitions (199/223 for VIP, 152/177 for MSTl), we randomly removed some 1035 repetitions; for neurons recorded for $<10$ repetitions (23/223 for VIP, 24/177 for MSTl), we 1036 filled in data by sampling with replacement. Once this was done, we computed the covariance 1037 matrix using the 'cov()' function in Matlab, as though all neurons had been recorded 1038 simultaneously. Since most pairs of neurons were not recorded simultaneously (simultaneous 1039 pairs: 226/24753 for VIP, 187/15576 for MSTl), the off-diagonal elements of the resulting 1040 covariance matrix do not reflect correlated noise for the vast majority of neuron pairs. However, 1041 the off-diagonal elements are generally non-zero since they reflect covariance that is driven by 1042 stimulus variations, and which is also dependent on the similarity of tuning properties of a pair.

1043 Separate covariance matrices were computed for leftward and rightward object direction classes

1044 and were averaged to get the covariance matrix used in Eqn. $5, \Sigma=\frac{1}{2} \cdot\left(\sum_{R}+\Sigma_{L}\right)$. However,

1045 results were very similar if a single covariance matrix was used for both object direction classes.

1046 We also compared our results to performance of a standard decoder based on logistic regression 104732,57 , which was trained on data and does not require explicit computation of a covariance matrix.

1048 Cross-validated output of the logistic regression decoder produced nearly identical results 1049 (Extended Data Fig. 5). 
1050 We took multiple approaches to decoding object direction from VIP and MSTl responses.

1051 1) Separate decoders for each task reference frame. In this approach, we trained separate

1052 decoders to classify object direction in world or head coordinates for each brain area. This

1053 approach assumes that the animal could have learned to read out VIP or MSTl activity in

1054 different ways for each task reference frame. For each task condition (head vs. world), FLD

1055 parameters $\left(w_{\text {world }}, k_{\text {world }}\right.$ and $\left.w_{\text {head }}, k_{\text {head }}\right)$ were computed separately from neural responses

1056 recorded in the corresponding task condition. Otherwise, each decoder was trained to report

1057 object direction across all stimulus conditions, including both self-motion directions and both

1058 Object + Visual and Object+Combined conditions. For each decoder, we randomly sampled 20

1059 trials (with replacement) from each neuron. 80\% of these trials were used for computing the

1060 classifier parameters as described above, and the remaining 20\% were used for computing

1061 classifier performance (fivefold cross-validation approach). This was repeated 1000 times and

1062 overall performance was found by averaging the results. For sliding window analyses, this

1063 resampling approach was repeated 100 times for each time bin.

1064 2) Common decoder for both reference frame conditions. We also investigated whether a

1065 single decoder with one set of common weights could correctly classify object direction in both

1066 head and world coordinates. This decoder examines the hypothesis that VIP or MSTl responses

1067 are modulated by self-motion signals in a task-dependent manner that allows for the same

1068 readout weights to be used for computing object direction in either head or world coordinates.

1069 For this analysis, FLD parameters were computed from neural responses that were recorded in

1070 both the head- and world-coordinate task conditions, as well as across both self-motion

1071 directions and both Object+Visual and Object+Combined conditions. All other aspects of the

1072 computation (e.g., cross-validation) were as described above for the separate decoders. 
1074 object and background-dot motion (in screen coordinates) under both task reference frames, we 1075 could test how well a decoder trained to perform the task in a particular reference frame would

1076 perform when supplied with neural responses from the other task reference frame condition.

1077 Specifically, for the cross-task decoders, we trained a decoder to perform the world coordinate

1078 task based on neural responses from the head coordinate task, and we trained a decoder to

1079 perform the head coordinate task using responses from the world task. All other aspects of the

1080 decoding procedure were as described above. This approach allowed us to test how well the

1081 neural representations in VIP or MSTl could generalize across tasks.

\section{Statistics and reproducibility}

In cases where the data met assumptions of normality, as assessed by Lilliefors test,

1085 parametric statistical tests were used, including t-tests, paired t-tests, and Pearson correlations.

1086 When data were not normally distributed, we used non-parametric tests, including the Wilcoxon

1087 rank sum test and the Wilcoxon signed rank test (for paired data).

1088 No statistical methods were used to pre-determine sample sizes but our sample sizes are 1089 comparable to, if not greater than, those reported in previous publications of a similar nature ${ }^{37,52,}$

$1090 \quad 53$. Data collection and analysis were not performed blind to the conditions of the experiments.

1091 However, all experimental conditions followed a standard protocol for each recording site and

1092 were entirely under computer control. Within each recording session, all stimulus conditions

1093 were block-randomized, such that the distinct stimuli were presented in a random order for each

1094 repetition. No animals were excluded from the analysis. Neurons were selected for analysis only 
1095 based on their receptive field location (as described above), and if they could be recorded for at

1096 least 3 stimulus repetitions in the main experiment.

1097

1098

1099 Code availability. Custom analysis code was written using MATLAB (v. 2018a). Matlab scripts

1100 employed are available from the corresponding author upon reasonable request.

1101

1102 Data availability: The data that support the findings of this study are available from the

1103 corresponding author upon reasonable request.

1105 51. Gu, Y., Watkins, P.V., Angelaki, D.E. \& DeAngelis, G.C. Visual and nonvisual 1106 contributions to three-dimensional heading selectivity in the medial superior temporal area. $J$ 1107 Neurosci 26, 73-85 (2006).

1108 52. Fetsch, C.R., Pouget, A., DeAngelis, G.C. \& Angelaki, D.E. Neural correlates of 1109 reliability-based cue weighting during multisensory integration. Nat Neurosci 15, 146-154 1110 (2012).

1111 53. Gu, Y., Angelaki, D.E. \& DeAngelis, G.C. Neural correlates of multisensory cue 1112 integration in macaque MSTd. Nat Neurosci 11, 1201-1210 (2008).

1113 54. Chen, A., DeAngelis, G.C. \& Angelaki, D.E. Representation of vestibular and visual cues 1114 to self-motion in ventral intraparietal cortex. J Neurosci 31, 12036-12052 (2011).

1115 55. Chen, A., DeAngelis, G.C. \& Angelaki, D.E. Macaque parieto-insular vestibular cortex: 1116 responses to self-motion and optic flow. J Neurosci 30, 3022-3042 (2010).

1117 56. Chen, A., Gu, Y., Takahashi, K., Angelaki, D.E. \& DeAngelis, G.C. Clustering of self1118 motion selectivity and visual response properties in macaque area MSTd. J Neurophysiol 100, 1119 2669-2683 (2008).

1120 57. Bishop, C.M. Pattern Recognition and Machine Learning (Springer, New York, 2006). 


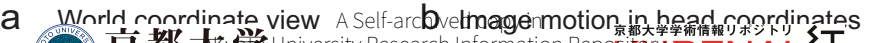

2iin 京都

fast
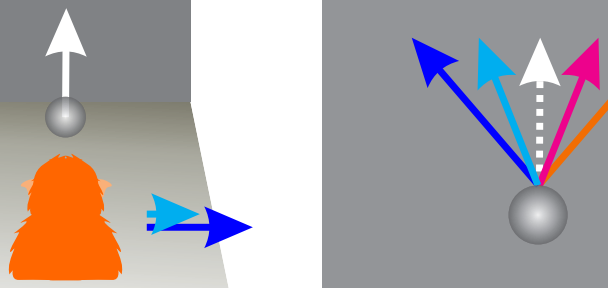


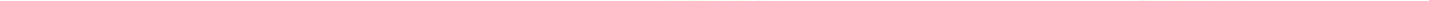


Object + Combined condition

World coordinate task: - - Right self-motion, - - -- Left self-motion

Head coordinate task: $-\square-$ Right self-motion, - $\square-$ - Left self-motion

Object + Visual condition

World coordinate task: -

- Right self-motion,

- Left self-motion

Head coordinate task:

- Right self-motion, - - $\square$-- Left self-motion

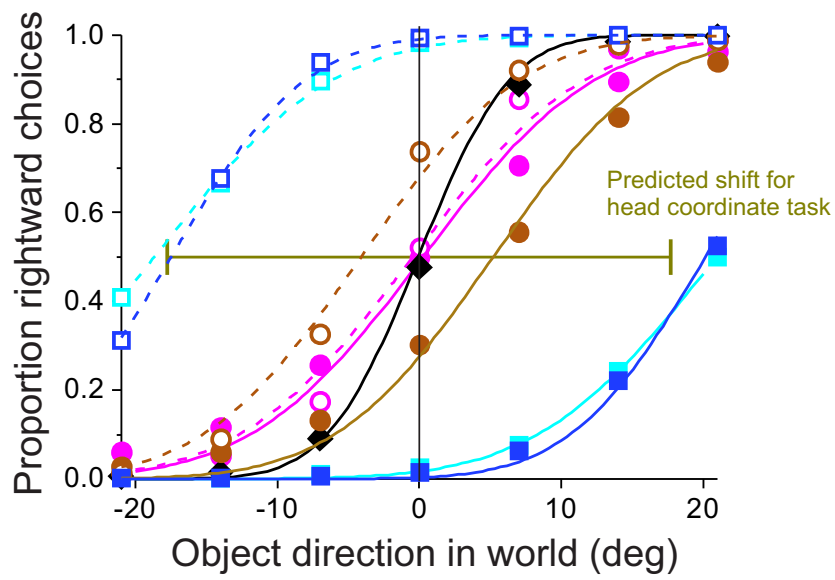

bu. World coordinate task
$\square \quad$ Monkey N
Monkey K
- Mean

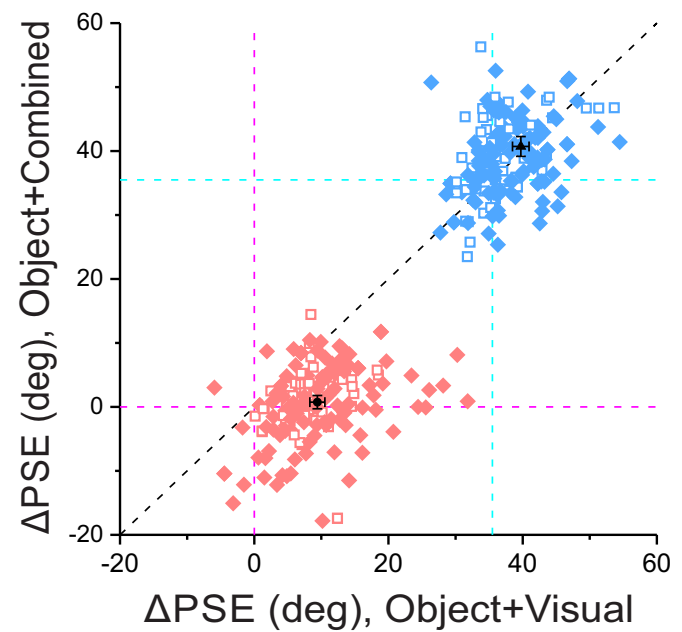

- Monkey N

- Monkey K

- Mean 
Object + Combined coindition

World-coordinate task: - - Right self-motion, -- $\bigcirc$ - Left self-motion

Head-coordinate task:

Right self-motion,

Left self-motion

a
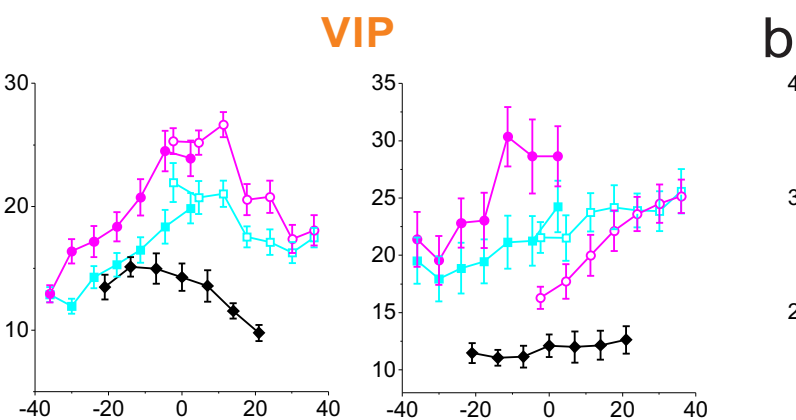

b

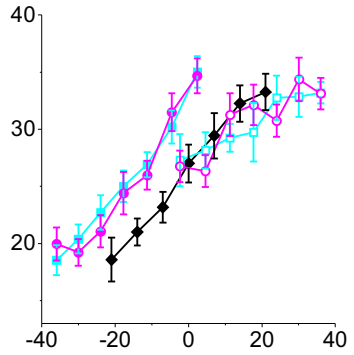

MSTI

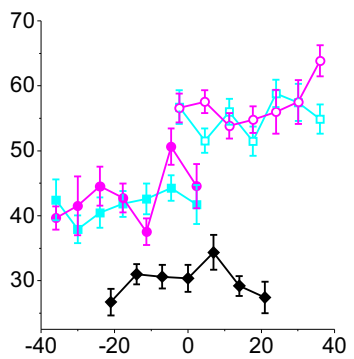

Object direction in head coordinates (deg)
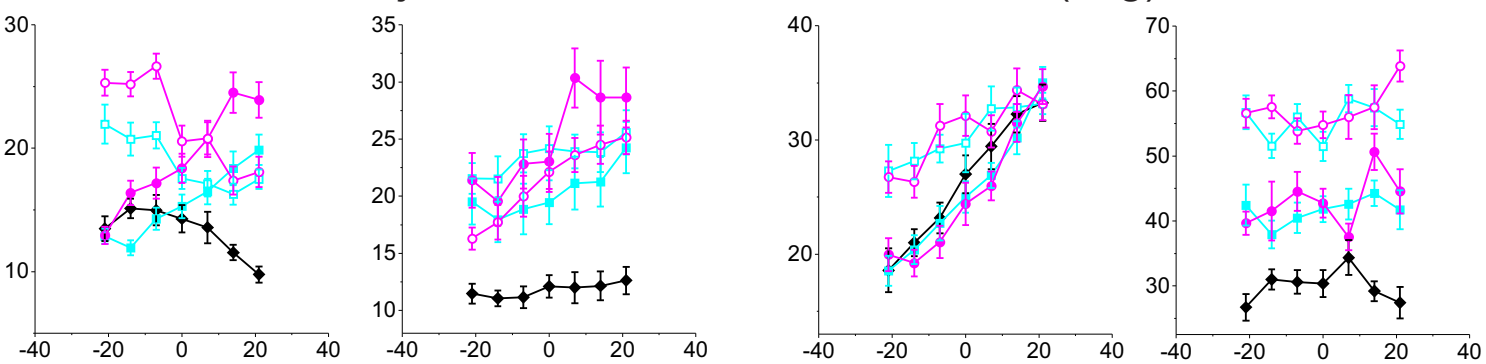

Object direction in world coordinates (deg)

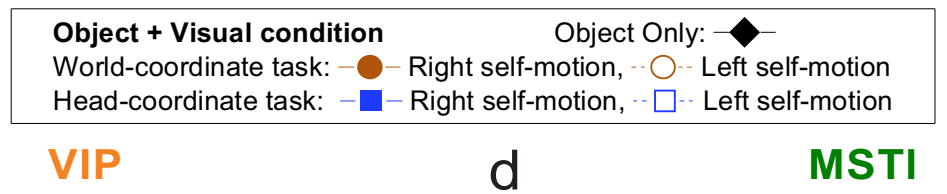

C
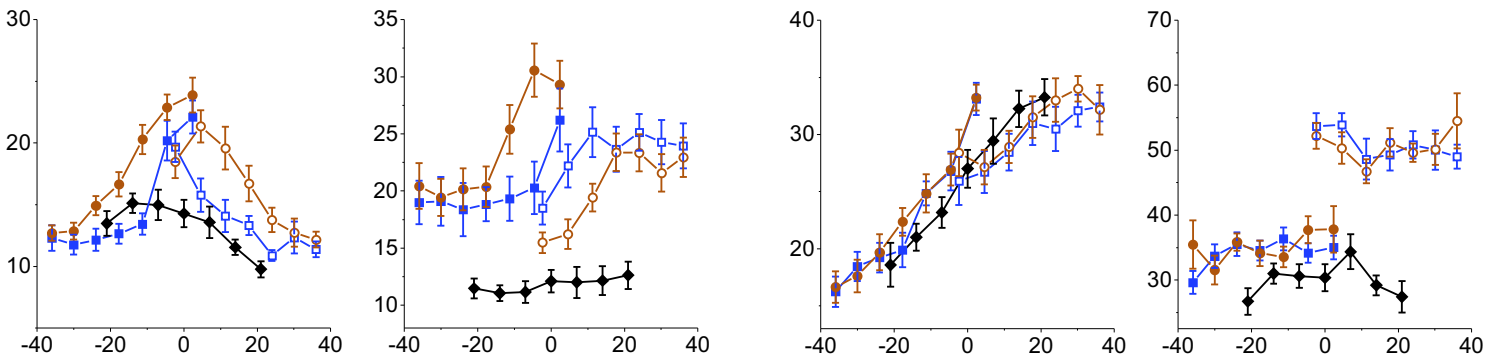

Object direction in head coordinates (deg)
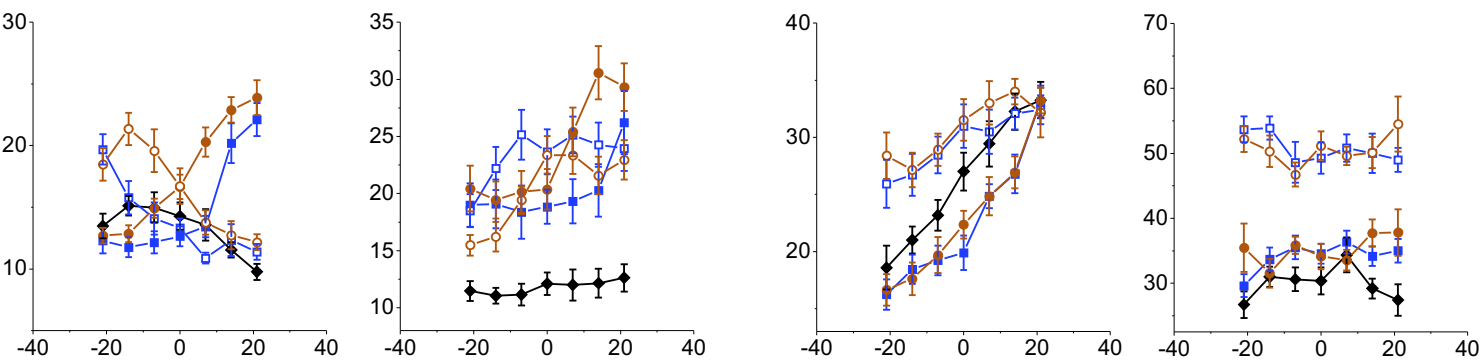

Object direction in world coordinates (deg) 


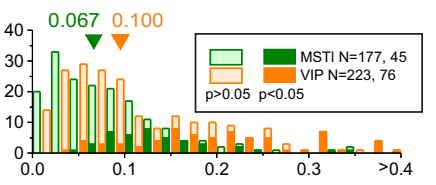

Object+Combined

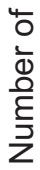

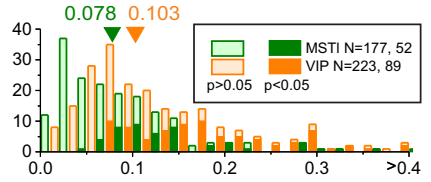

Modulation index

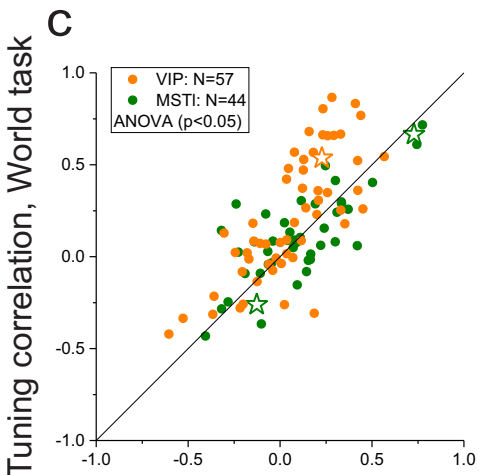

Tuning correlation, Head task
京都大学学術情報リポジトリ

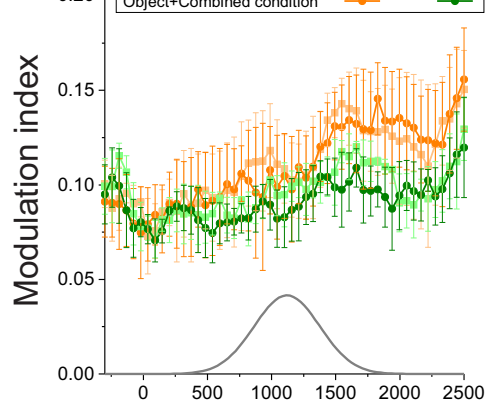

Time from stimulus onset (ms)

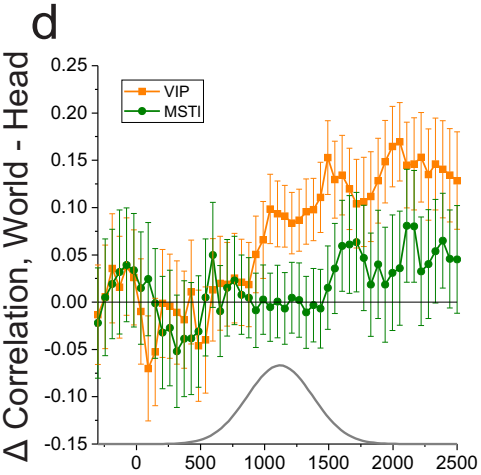

Time from stimulus onset (ms) 


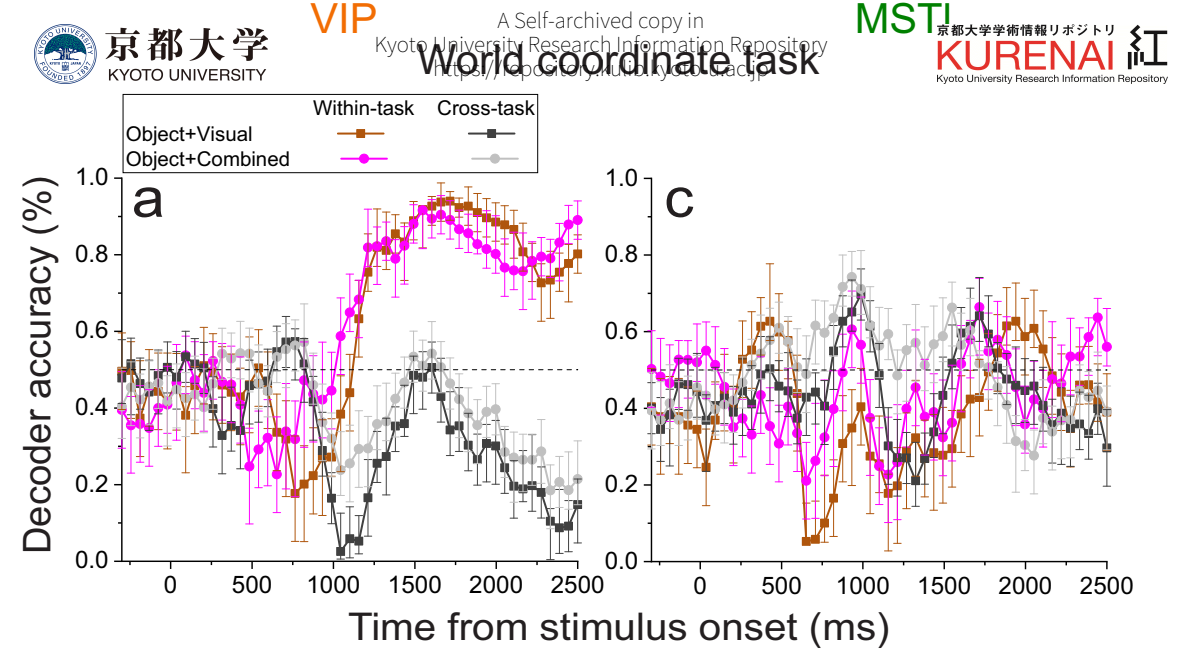

Head coordinate task

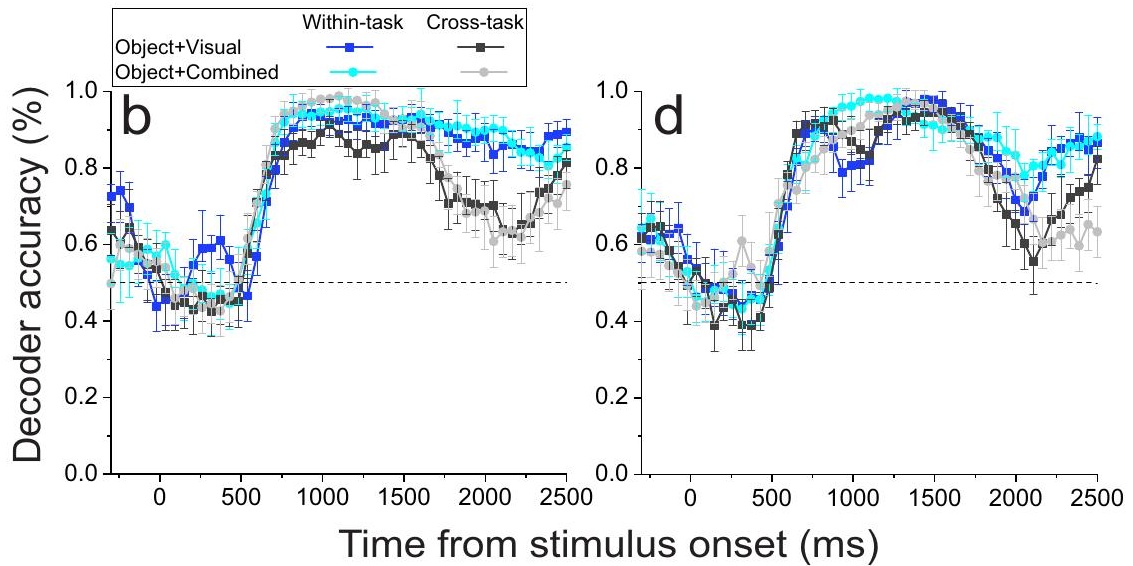


A Self-archived copy in

京都大学 Kyoto University Research Information Repository KYOTO UNIVERSITY https://repository.kulib.kyoto-u.ac.jp

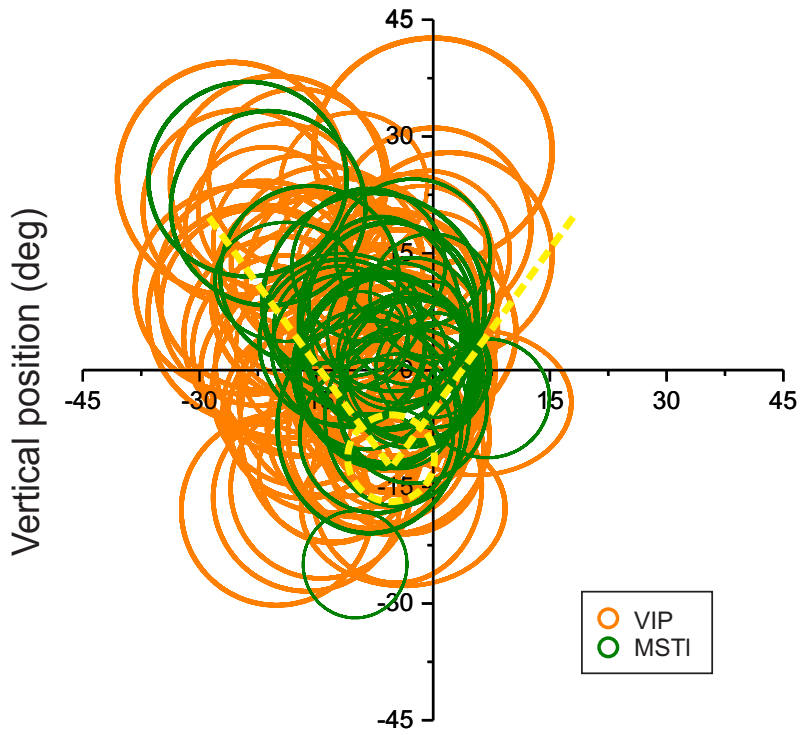

Horizontal position (deg) 
Head coordinate task: - - - Right self-motion, - - $\square$ - - Left self-motion

Object + Visual condition

World coordinate task: - - Right self-motion, - - ○-- Left self-motion

Head coordinate task: - - - Right self-motion, - - $\square$-- Left self-motion

\section{Object + Combined condition}
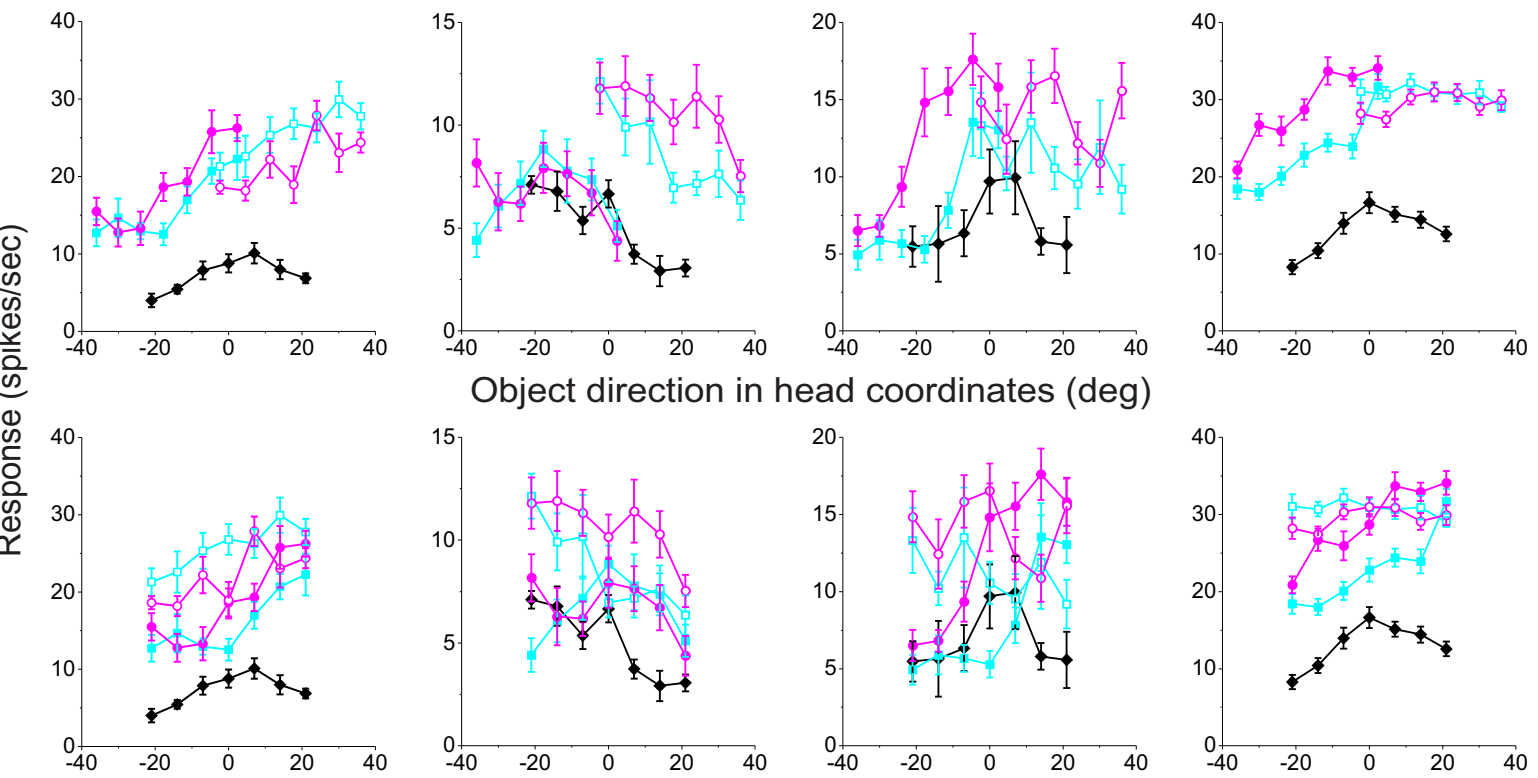

Object direction in head coordinates (deg)
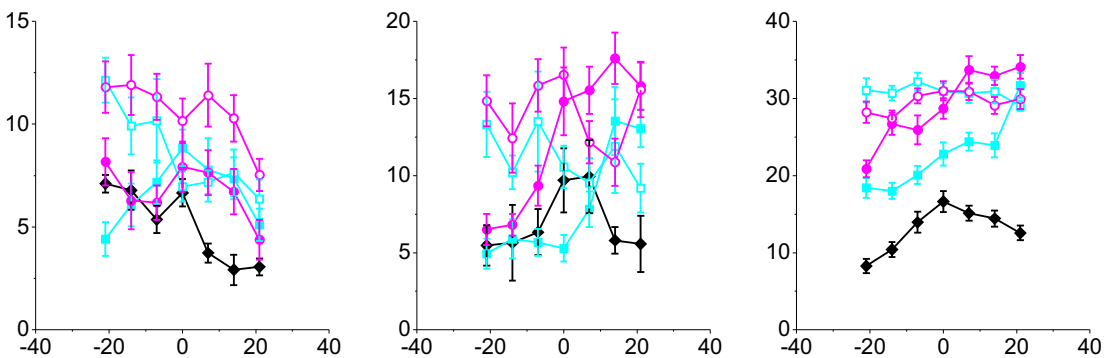

Object direction in world coordinates (deg)

\section{Object + Visual condition}
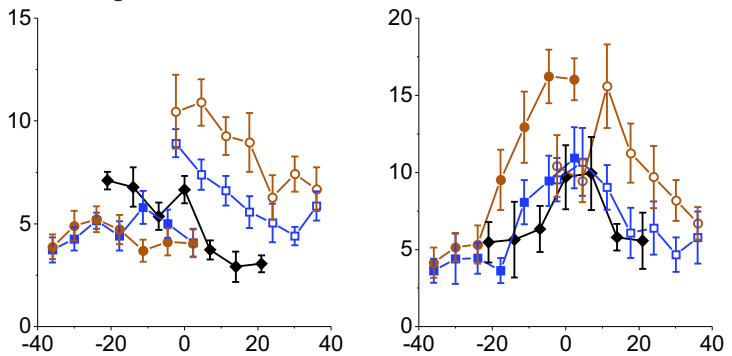

Object direction in head coordinates (deg)
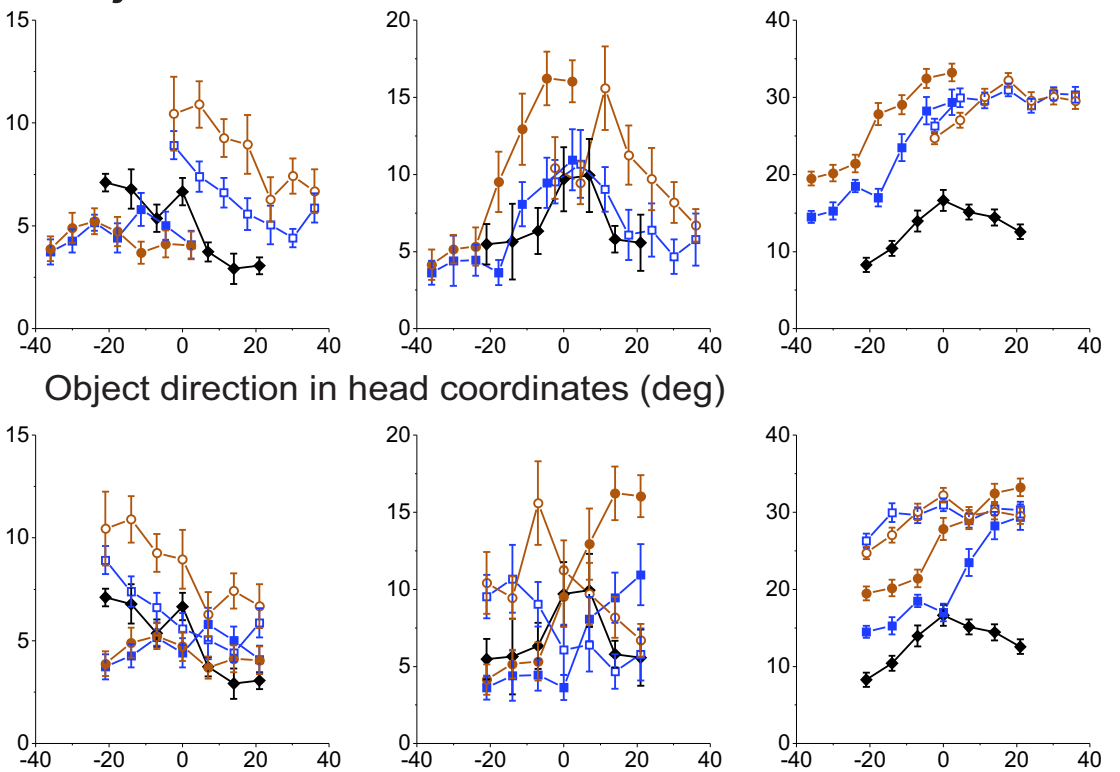

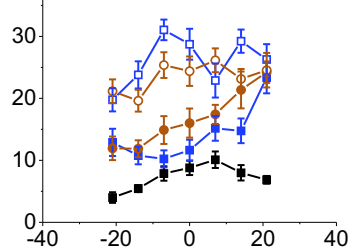



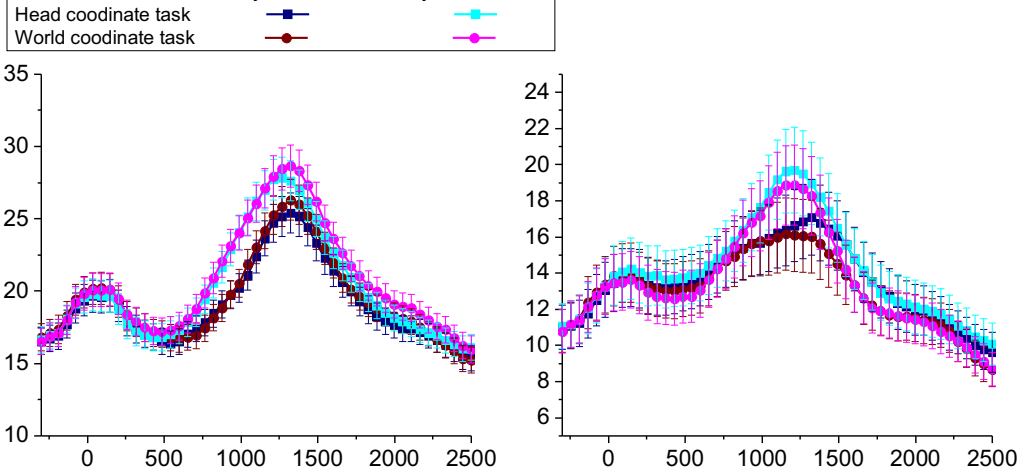

\section{Time from stimulus onset (ms)}
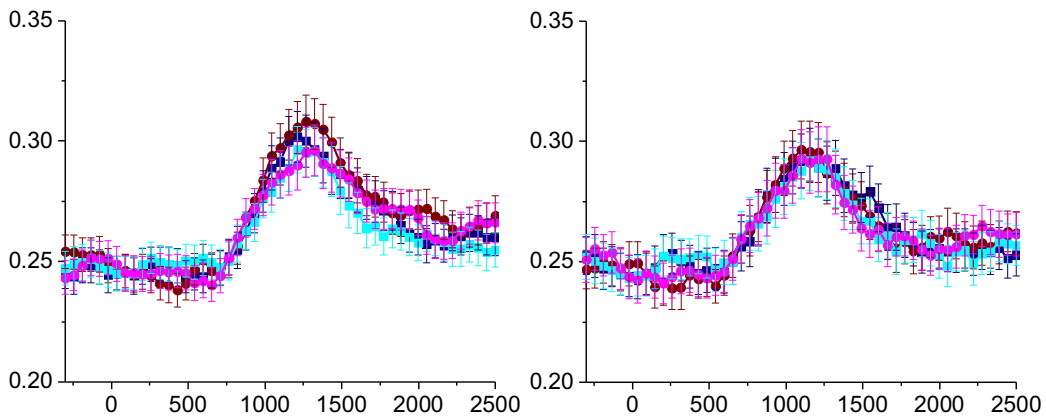

Time from stimulus onset (ms) 
A Self-archived copy in

京都大学

i. KYOTO UNIVERSITY
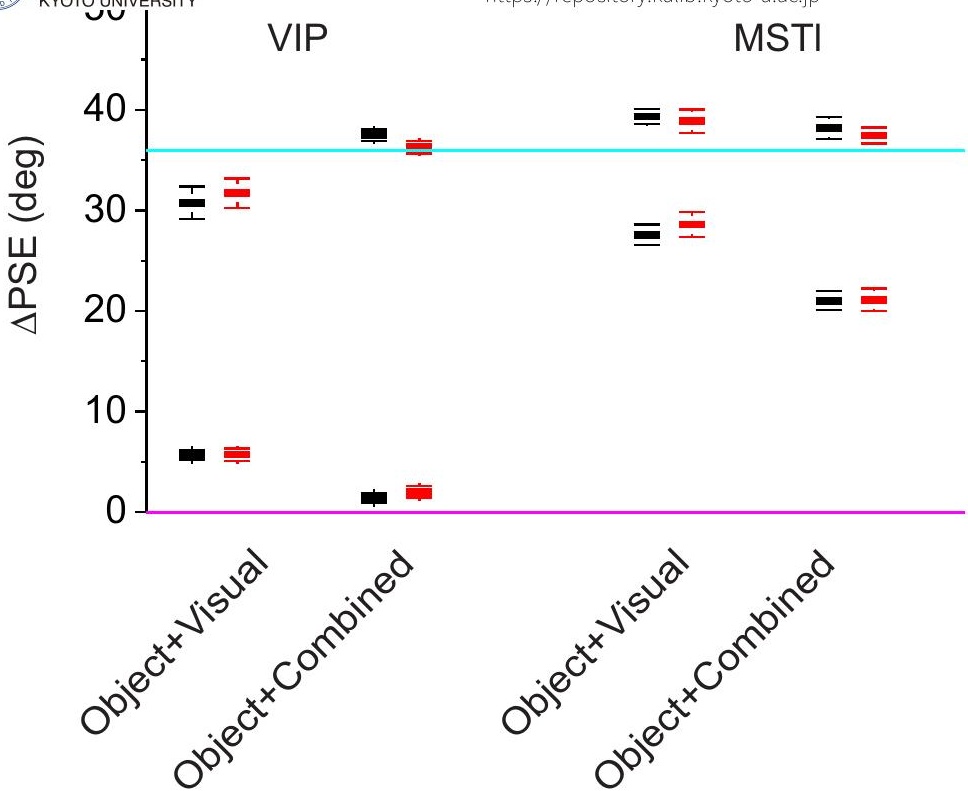
KURENAII 紅 KURENAIII
Predicted shift for

head coordinate task

- FLD

- Logistic regression
Predicted shift for world coordinate task 
京都大学 dition

KYOTO UNIVERSITY-O-Right self-motion, $\cdot \bigcirc \cdot$ - Left self-motion Head coordinate task: - - Right self-motion, - $\square$. Left self-motion

Object + Visual condition

World coordinate task:

Head coordinate task:

- Right self-motion, $\cdot \cdot \bigcirc \cdots$ Left self-motion - Right self-motion, - - $\square$ - Left self-motion

VIP

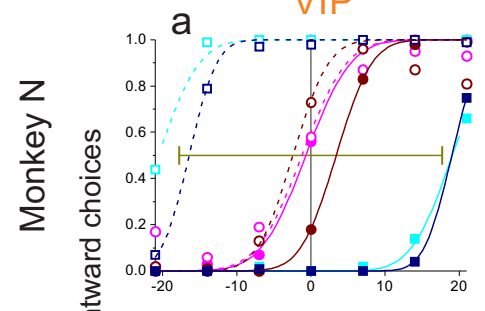

$\frac{\searrow}{\partial}$

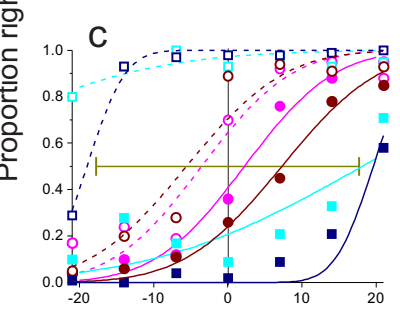

Object direction in world coordinates (deg)

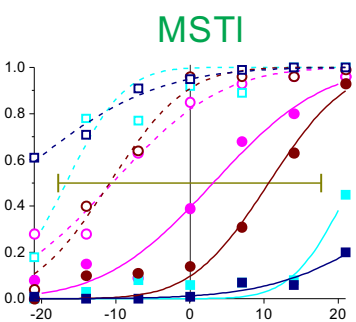

Separate decoders

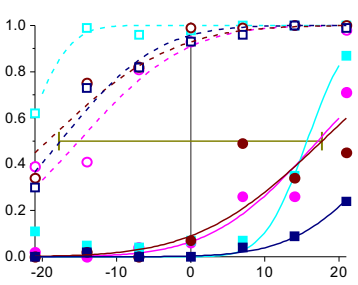

$\triangle$ PSE (deg), Object+Visual
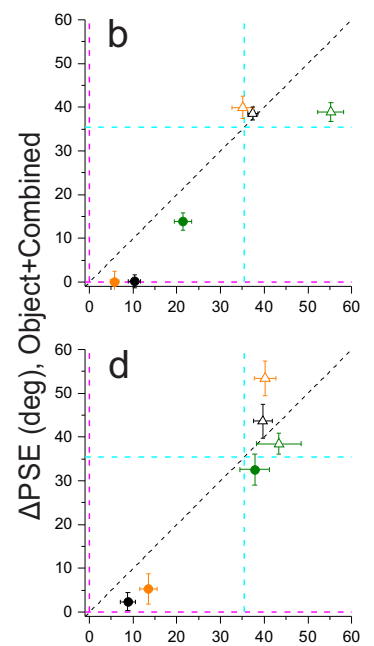
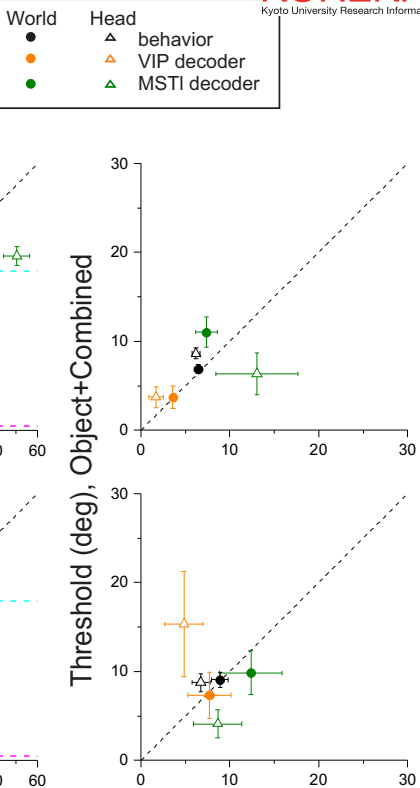

Threshold (deg), Object+Visual

\section{Single decoder}
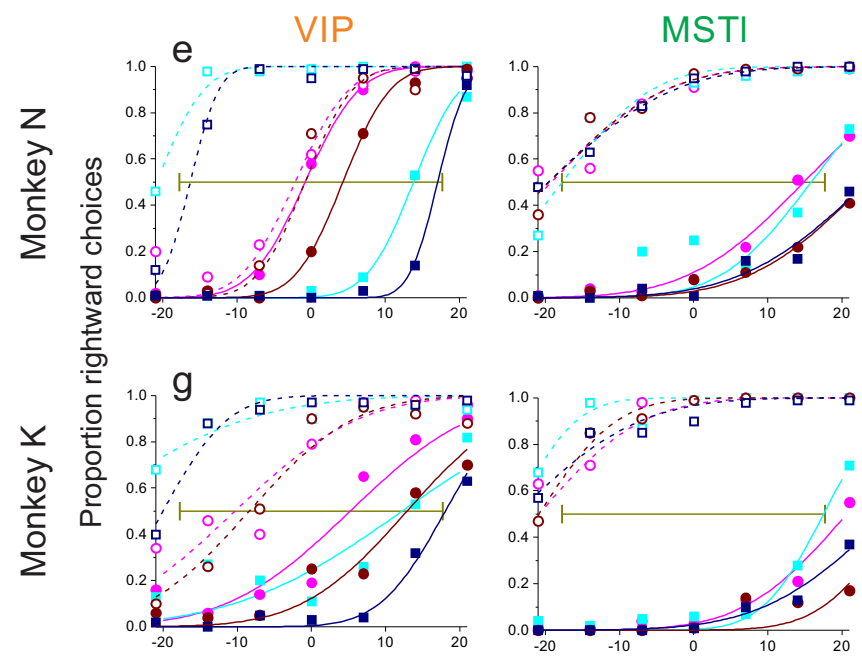

Object direction in world coordinates (deg)
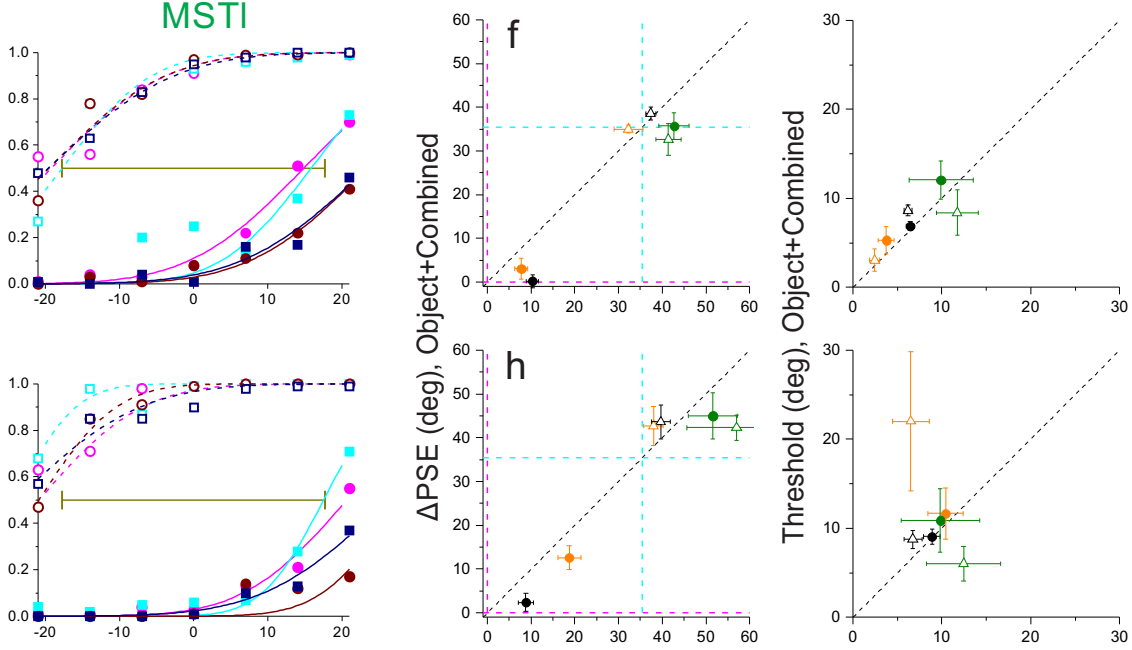

$\triangle \mathrm{PSE}$ (deg), Object+Visual

Threshold (deg), Object+Visual 


\section{京都大学— VIP A Self-archived copy in}

MST| 京都大学掌㭪情豭リポジトリく
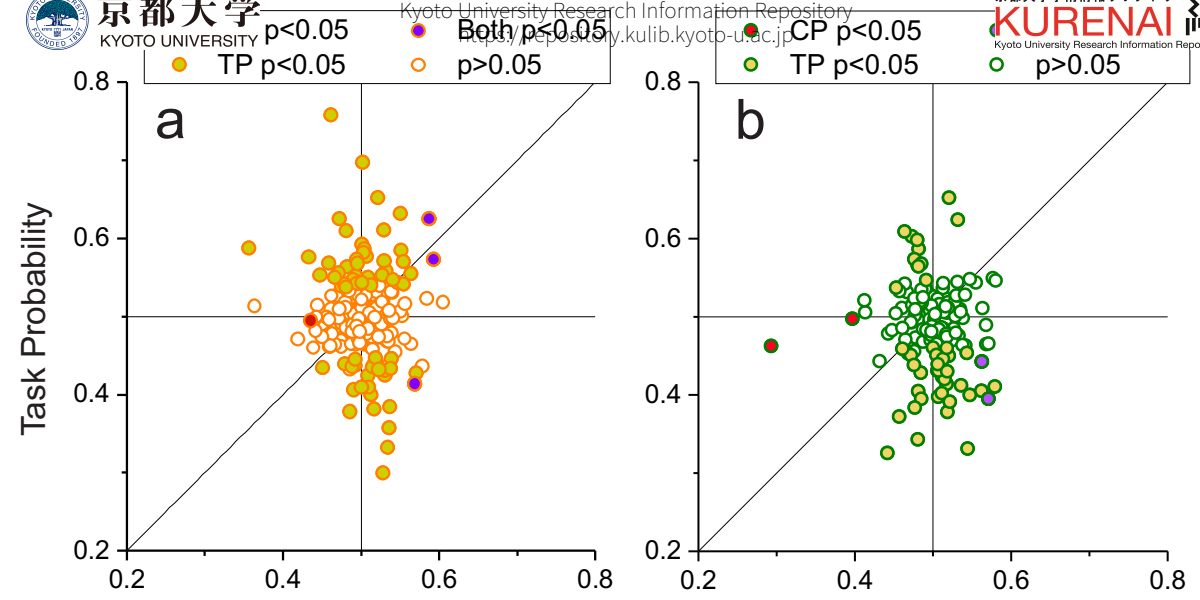

Choice Probability, choice effect removed

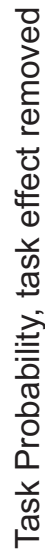
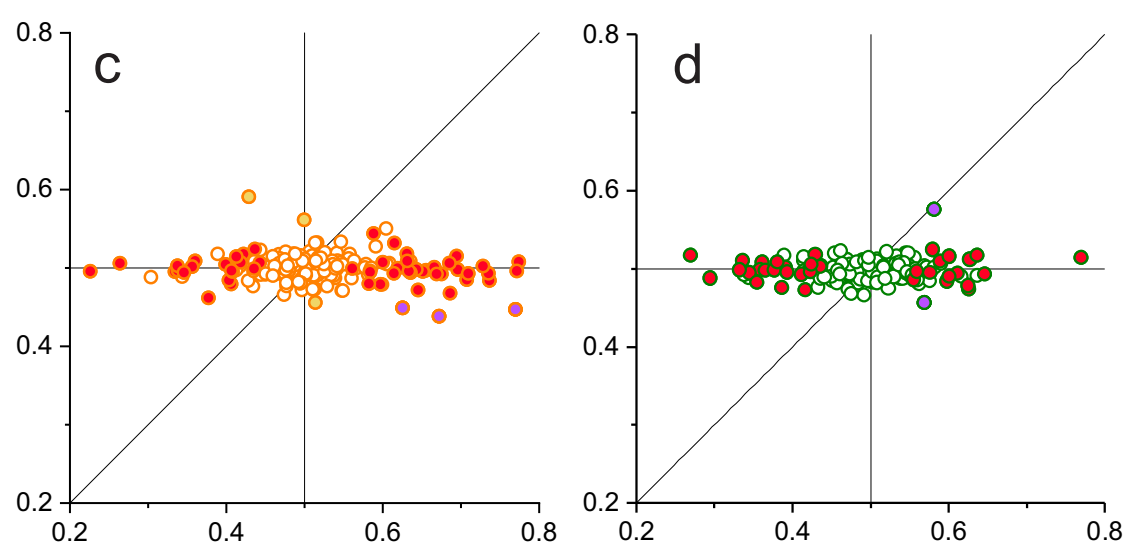

Choice Probability

Task effect removed

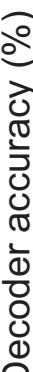

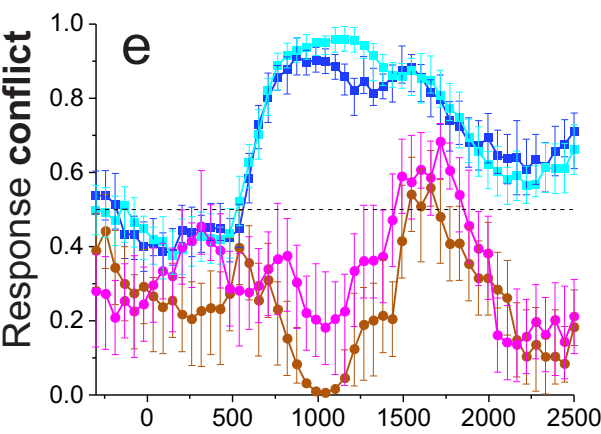

Choice effect removed

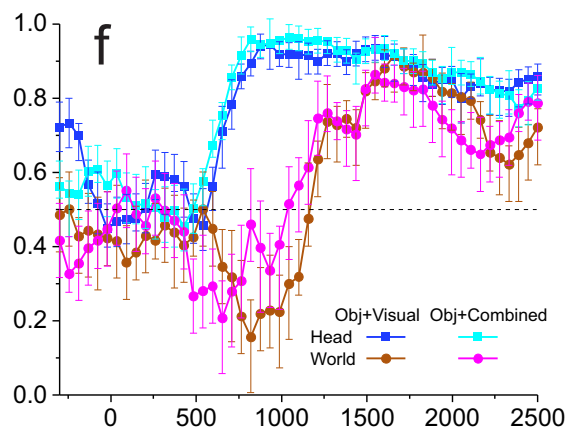




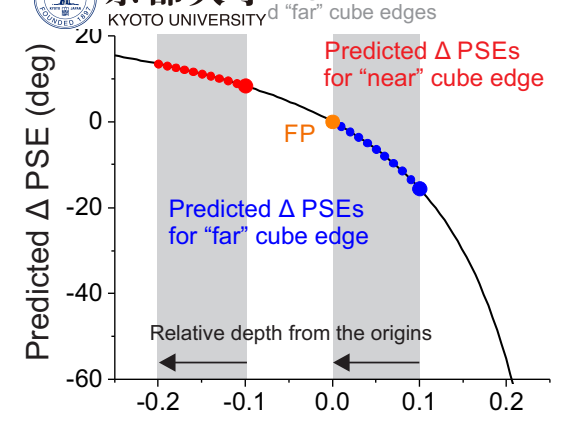

Depth of the cube edge $(\mathrm{m})$

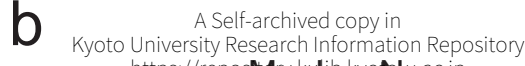

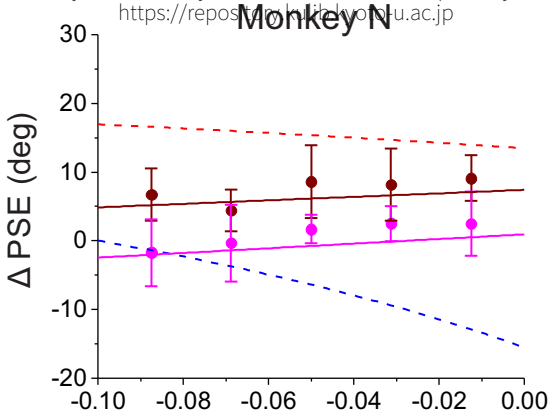

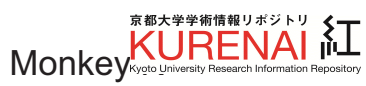

Relative depth from the origins (m) 


\section{Inventory of Supporting Information}

\section{Manuscript \#: NN-A69354B}

Corresponding author name(s): Ryo Sasaki

\section{Instructions:}

Please complete each of the Inventory Tables below to outline your Extended Data and Supplementary Information items.

There are four sections; 1. Extended Data, 2A. Supplementary Information: Flat Files, 2B. Supplementary Information: Additional Files, and 3. Source Data. Each section includes specific instructions. Please complete these tables as fully as possible. We ask that you avoid using spaces in your file names, and instead use underscores, i.e.: Smith_ED_Fig1.jpg not Smith ED Fig1.jpg

Please note that titles and descriptive captions will only be lightly edited, so please ensure that you are satisfied with these prior to submission.

If you have any questions about any of the information contained in this inventory, please contact the Editorial Assistant: neurosci@us.nature.com 


\section{Extended Data}

\section{Complete the Inventory below for all Extended Data figures.}

- Keep Figure Titles to one sentence only

- File names should include the Figure Number. i.e.: Smith_ED Fig1.jpg

- Please be sure to include the file extension in the Filename. Note that Extended Data files must be submitted as .jpg, .tif or .eps files only

- All Extended Data figure legends must be provided in the Inventory below and should not exceed 300 words each (if possible)

- Please include Extended Data ONLY in this table

\begin{tabular}{|l|l|l|l|}
\hline Figure \# & $\begin{array}{l}\text { Figure title } \\
\text { One sentence only }\end{array}$ & $\begin{array}{l}\text { Filename } \\
\text { This should be the } \\
\text { name the file is saved as } \\
\text { when it is uploaded to } \\
\text { our system. Please } \\
\text { include the file } \\
\text { extension. i.e.: } \\
\text { Smith_ED_Fig1.jpg }\end{array}$ & $\begin{array}{l}\text { Figure Legend } \\
\text { If you are citing a reference for the first time in these legends, } \\
\text { please include all new references in the Online Methods References } \\
\text { section, and carry on the numbering from the main References } \\
\text { section of the paper. }\end{array}$ \\
\hline Extended Data Fig. 1 & $\begin{array}{l}\text { Summary of } \\
\text { psychophysical } \\
\text { thresholds (inverse of } \\
\text { sensitivity) across task } \\
\text { conditions. }\end{array}$ & $\begin{array}{l}\text { Sasaki_ED_Fig1.eps } \\
\text { (n) }\end{array}$ & $\begin{array}{l}\text { Extended Data Figure 1. Summary of psychophysical } \\
\text { thresholds (inverse of sensitivity) across task conditions. (a) } \\
\text { Average threshold for the Object Only condition (no self- } \\
\text { motion) is plotted against average thresholds for the } \\
\text { Object+Visual and Object+Combined conditions for the world } \\
\text { (brown/magenta) and head (blue/cyan) coordinate tasks. } \\
\text { Error bars represent 95\% confident intervals. Averages taken } \\
\text { over n=185 sessions across the two animals. (b) For each } \\
\text { session, threshold in the Object+Combined condition is } \\
\text { plotted against the corresponding threshold in the } \\
\text { Object+Visual condition. Black symbols show mean thresholds }\end{array}$ \\
\hline
\end{tabular}




\begin{tabular}{|c|c|c|c|}
\hline & & & $\begin{array}{l}\text { and error bars represent } 95 \% \text { confidence intervals. Data from } \\
128 \text { sessions for Monkey N and } 57 \text { sessions for monkey K. }\end{array}$ \\
\hline Extended Data Fig. 2 & $\begin{array}{l}\text { Summary of receptive } \\
\text { field locations for } \\
\text { populations of VIP } \\
\text { (orange, } \mathrm{N}=66 \text { ) and } \\
\text { MSTI (green, } \mathrm{N}=44 \text { ) } \\
\text { neurons. }\end{array}$ & Sasaki_ED_Fig2.eps & $\begin{array}{l}\text { Extended Data Figure } 2 \text {. Summary of receptive field locations } \\
\text { for populations of VIP (orange, N=66) and MSTI (green, } N=44 \text { ) } \\
\text { neurons. Cells are included here if they had significant } \\
\text { structure in receptive field maps obtained by reverse } \\
\text { correlation ( } 17 \% \text { of VIP and } 13 \% \text { of MSTI neurons) or if they } \\
\text { had clear hand-mapped receptive fields for which good } \\
\text { estimates of RF center and size were obtained ( } 13 \% \text { of VIP } \\
\text { neurons and } 12 \% \text { of MSTI neurons). Significant structure in } \\
\text { reverse correlation maps was assessed by a two-sided } \\
\text { permutation test ( }<<0.05 \text { ), in which we scrambled the } \\
\text { relationship between response amplitude and stimulus } \\
\text { location within the RF, as described previously } 56 \text {. Ellipses } \\
\text { approximate the RF dimensions and were derived either from } \\
\text { a two-dimensional Gaussian fit (contour at half-maximal } \\
\text { response) to receptive field maps obtained by reverse } \\
\text { correlation (VIP: N=38; MSTI: N=23), or from hand mapping } \\
\text { (VIP: N=28; MSTI: N=21). Coordinate }(0,0) \text { represents the } \\
\text { center of the visual display, where the fixation target was } \\
\text { located. Yellow dashed lines represent the starting location of } \\
\text { the moving object and the range of directions in head } \\
\text { coordinates. }\end{array}$ \\
\hline Extended Data Fig. 3 & $\begin{array}{l}\text { Data from four } \\
\text { additional VIP neurons, } \\
\text { illustrating diversity of } \\
\text { effects of self-motion on } \\
\text { tuning curves. }\end{array}$ & Sasaki_ED_Fig3.eps & $\begin{array}{l}\text { Extended Data Figure 3. Data from four additional VIP } \\
\text { neurons, illustrating diversity of effects of self-motion on } \\
\text { tuning curves. Top: Object+Combined condition. Bottom: } \\
\text { Object+Visual condition. Format as in Fig. } 4 \text {. Error bars denote } \\
\text { SEM ( } n=10 \text { stimulus repetitions per datum). }\end{array}$ \\
\hline Extended Data Fig. 4 & $\begin{array}{l}\text { Summary of time } \\
\text { courses of average firing } \\
\text { rates and directional } \\
\text { selectivity. }\end{array}$ & Sasaki_ED_Fig4.eps & $\begin{array}{l}\text { Extended Data Figure 4. Summary of time courses of average } \\
\text { firing rates and directional selectivity. (a) Average response } \\
\text { across all } 223 \text { VIP and } 177 \text { MSTI neurons is shown for each } \\
\text { stimulus condition for both the head and world coordinate } \\
\text { task conditions. For each neuron, responses were taken from } \\
\text { the object motion direction that elicited the maximum firing }\end{array}$ \\
\hline
\end{tabular}




\begin{tabular}{|c|c|c|c|}
\hline & & & $\begin{array}{l}\text { rate. Error bars represent SEM. Color coding as in Fig. } 7 \text {. } \\
\text { Results were nearly identical if the responses of neurons were } \\
\text { normalized before averaging. (b) Average direction } \\
\text { discrimination index (DDI) for populations of VIP ( } n=223 \text { ) and } \\
\text { MSTI ( } n=177 \text { ) neurons (see Methods, Eqn. 2). DDI values were } \\
\text { computed separately for leftward and rightward self-motion } \\
\text { and then averaged for each neuron. Error bars represent } 95 \% \\
\text { confidence intervals. For this figure, both average responses } \\
\text { and DDI values were computed within a } 300 \text { ms sliding time } \\
\text { window that was advanced across the stimulus epoch in steps } \\
\text { of } 50 \mathrm{~ms} \text {. }\end{array}$ \\
\hline Extended Data Fig. 5 & $\begin{array}{l}\text { Decoder results are } \\
\text { robust to the type of } \\
\text { classifier used. }\end{array}$ & Sasaki_ED_Fig5.eps & $\begin{array}{l}\text { Extended Data Figure } 5 \text {. Decoder results are robust to the } \\
\text { type of classifier used. Black data points represent results } \\
\text { from the FLD classifier used in all main figures. Red data points } \\
\text { show results from a logistic regression decoder. For this } \\
\text { comparison, the same population responses were used for } \\
\text { training and testing each decoder. The results are very robust } \\
\text { to the type of decoder used. Error bars represent } 95 \% \\
\text { confidence intervals (across } n=1000 \text { bootstraps). }\end{array}$ \\
\hline Extended Data Fig. 6 & $\begin{array}{l}\text { Comparison of decoder } \\
\text { results across animals. }\end{array}$ & Sasaki_ED_Fig6.eps & $\begin{array}{l}\text { Extended Data Figure } 6 \text {. Comparison of decoder results } \\
\text { across animals. (a-d) Results for separate decoders trained to } \\
\text { perform the world and head coordinate tasks. Format as in } \\
\text { Figure } 6 \text {. Each row shows results separately for each animal. } \\
\text { Pink and cyan dashed lines in panels } b \text { and } d \text { : expected } \Delta \text { PSE } \\
\text { for perfect performance in the world and head coordinate } \\
\text { tasks, respectively. Error bars in panels b and d represent } 95 \% \\
\text { confidence intervals (across } n=1000 \text { bootstraps). (e-h) Results } \\
\text { for the single decoder, shown separately for each animal. } \\
\text { Decoders were trained separately using responses from each } \\
\text { animal, yet main results are conserved across subjects. Error } \\
\text { bars represent } 95 \% \text { confidence intervals (across } n=1000 \\
\text { bootstraps). Format as in panels a-d. }\end{array}$ \\
\hline Extended Data Fig. 7 & $\begin{array}{l}\text { Effect of partial cube } \\
\text { frame on single-unit }\end{array}$ & Sasaki_ED_Fig7.eps & $\begin{array}{l}\text { Extended Data Figure } 7 \text {. Effect of partial cube frame on } \\
\text { single-unit responses and population decoding. }(a, d)\end{array}$ \\
\hline
\end{tabular}




\begin{tabular}{|c|c|c|c|}
\hline & $\begin{array}{l}\text { responses and } \\
\text { population decoding. }\end{array}$ & & $\begin{array}{l}\text { Distributions of the cube effect index (CEI, see Methods) for } \\
\text { areas VIP and MSTI, respectively, in the world coordinate task. } \\
\text { Black and gray shading denotes neurons with CEI values that } \\
\text { are significantly different from zero and non-significant, } \\
\text { respectively (two-sided permutation test, } p<0.05 \text { ). (b, e) } \\
\text { Distributions of CEI for VIP and MSTI, respectively, in the head } \\
\text { coordinate task condition. (c, f) Distributions of the difference } \\
\text { in CEI ( } \triangle C E I \text { ) between world and head task conditions for VIP } \\
\text { and MSTI, respectively. Green and purple shading indicates a } \\
\text { median split of the data based on the absolute value, | } \Delta C E I \mid \text {. } \\
\text { (g, h) Comparison of decoder accuracy (proportion correct) for } \\
\text { populations of neurons with above-median | } \Delta \text { CEI| (abscissa) } \\
\text { and below-median | } \triangle \text { CEI| (ordinate) values, for areas VIP and } \\
\text { MSTI, respectively. Error bars represent } 95 \% \text { confidence } \\
\text { intervals (across } n=1000 \text { bootstraps). Data in these panels } \\
\text { come from decoders that were trained separately for the } \\
\text { world and head coordinate task conditions. (i, j) Same as } \\
\text { panels g and h, except for a single decoder trained to perform } \\
\text { the task across both reference frame conditions. Format as in } \\
\text { g, h. }\end{array}$ \\
\hline Extended Data Fig. 8 & $\begin{array}{l}\text { Summary of choice- } \\
\text { related and task-related } \\
\text { response modulations. }\end{array}$ & Sasaki_ED_Fig8.eps & $\begin{array}{l}\text { Extended Data Figure 8. Summary of choice-related and task- } \\
\text { related response modulations. (a) Scatter plot of task } \\
\text { probability (TP) and choice probability (CP) values for VIP } \\
\text { neurons ( } N=223 \text { ). Color of the symbol centers corresponds to } \\
\text { significance of TP and CP values as follows: blue center, both } \\
\text { TP and CP are significantly different from } 0.5 \text { (two-sided } \\
\text { permutation test, } p<0.05 \text { ); red center, only CP is significantly } \\
\text { different from } 0.5 \text {; gold center, only TP is significantly } \\
\text { different from } 0.5 \text {; white center, neither TP nor CP is } \\
\text { significant. The observation that TP and CP values are largely } \\
\text { uncorrelated here is an empirical observation that is not } \\
\text { enforced by the analysis. (b) Scatter plot of TP and CP values } \\
\text { for MSTI neurons ( } N=177) \text {. Symbol center color conventions as } \\
\text { in panel a. (c) Scatter plot of TP values for VIP neurons }\end{array}$ \\
\hline
\end{tabular}




\begin{tabular}{|c|c|c|c|}
\hline & & & $\begin{array}{l}\text { computed separately for right and left choices }(\mathrm{N}=223) \text {. ( } \mathrm{d}) \\
\text { Same as panel c but for MSTI neurons }(\mathrm{N}=177) \text {. (e) Scatter } \\
\text { plot comparing CP values from VIP for the world and head } \\
\text { coordinate task conditions ( } N=223) \text {. ( } f \text { ) Same as panel e but for } \\
\text { MSTI neurons ( } N=177) \text {. }\end{array}$ \\
\hline Extended Data Fig. 9 & $\begin{array}{l}\text { Effects of selectively } \\
\text { removing choice- or } \\
\text { task-related response } \\
\text { modulations. }\end{array}$ & Sasaki_ED_Fig9.eps & $\begin{array}{l}\text { Extended Data Figure 9. Effects of selectively removing } \\
\text { choice- or task-related response modulations. (a) Scatter plot } \\
\text { of TP and CP values for VIP ( } \mathrm{N}=223 \text { ) after selective removal of } \\
\text { choice-related response modulations (see Methods for } \\
\text { details). Format as in Extended Data Fig. 8a. (b) Same as panel } \\
\text { a except for MSTI ( } \mathrm{N}=177 \text { ). Format as in Extended Data Fig. } \\
\text { 8b. (c) Scatter plot of TP and CP values for VIP after selective } \\
\text { removal of task-related response modulations. (d) Same as } \\
\text { panel c, except for MSTI. (e) Time course of decoder } \\
\text { performance based on activity of } 223 \text { VIP neurons on } \\
\text { response conflict trials, after removal of task-related response } \\
\text { modulations. Data are shown for the case of separate } \\
\text { decoders for world and head coordinate task conditions. } \\
\text { Format as in Fig. } 7 \mathrm{~b} \text {. Error bars represent } 95 \% \text { confidence } \\
\text { intervals (across n=100 bootstraps). (f) Time course of VIP } \\
\text { decoder performance, as in panel e, but after removal of } \\
\text { choice-related response modulations. }\end{array}$ \\
\hline Extended Data Fig. 10 & $\begin{array}{l}\text { Results from behavioral } \\
\text { control sessions in } \\
\text { which the depth of the } \\
\text { partial cube was varied } \\
\text { across trials. }\end{array}$ & Sasaki_ED_Fig10.eps & $\begin{array}{l}\text { Extended Data Figure 10. Results from behavioral control } \\
\text { sessions in which the depth of the partial cube was varied } \\
\text { across trials. (a) Predicted } \triangle \text { PSE values are shown as a } \\
\text { function of the depth of the partial cube. Red and blue data } \\
\text { points show predicted } \triangle P S E \text { values and depths for the near } \\
\text { and far edges of the cube. (b) Dashed curves replot the } \\
\text { predictions from panel a, where the horizontal axis is now } \\
\text { depth relative to the origins for the near (red) and far (blue) } \\
\text { cube edges (where the origins are the farthest depths for each } \\
\text { edge). Data points represent behavioral } \triangle P S E \text { values for the } \\
\text { two monkeys ( } n=7 \text { sessions for each animal); magenta and } \\
\text { brown data points show results for the Object+Combined and }\end{array}$ \\
\hline
\end{tabular}




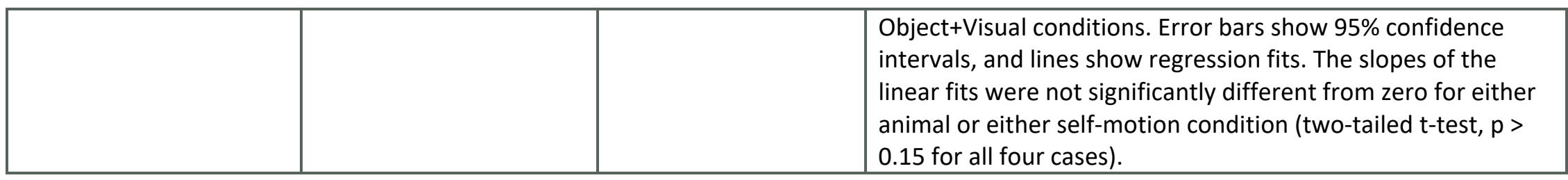

Delete rows as needed to accommodate the number of figures (10 is the maximum allowed).

\section{Supplementary Information:}

\section{A. Flat Files}

Complete the Inventory below for all additional textual information and any additional Supplementary Figures, which should be supplied in one combined PDF file.

- Row 1: A combined, flat PDF containing any Supplementary Methods, Discussion, Equations, Notes, Additional Supplementary Figures, simple tables, and all associated legends. Only one such file is permitted.

- Row 2: Nature Research's Reporting Summary; please provide an updated Summary, fully completed, without any mark-ups or comments. Please note that this is a required document.

\begin{tabular}{|c|c|c|c|}
\hline Item & Present? & $\begin{array}{l}\text { Filename } \\
\text { This should be the name } \\
\text { the file is saved as when it } \\
\text { is uploaded to our system, } \\
\text { and should include the file } \\
\text { extension. The extension } \\
\text { must be .pdf }\end{array}$ & $\begin{array}{l}\text { A brief, numerical description of file contents. } \\
\text { i.e.: Supplementary Figures 1-4, Supplementary Discussion, and } \\
\text { Supplementary Tables 1-4. }\end{array}$ \\
\hline Supplementary Information & No & & \\
\hline
\end{tabular}




\begin{tabular}{|l|l|l|}
\hline Reporting Summary & Yes & $\begin{array}{l}\text { Sasaki_nr-reporting- } \\
\text { summary_RENEWED }\end{array}$ \\
\hline
\end{tabular}

\section{B. Additional Supplementary Files}

Complete the Inventory below for all additional Supplementary Files that cannot be submitted as part of the Combined PDF.

- Do not list Supplementary Figures in this table (see section 2A)

- Where possible, include the title and description within the file itself

- Spreadsheet-based tables and data should be combined into a workbook with multiple tabs, not submitted as individual files.

- Please note that the $O N L Y$ allowable types of additional Supplementary Files are:
o Supplementary Tables
o Supplementary Audio
o NMR Data
o Computational Data
o Supplementary Videos
o Supplementary Data
o Cryo-EM Data
o Suppl. Software

\begin{tabular}{|l|l|l|l|}
\hline & $\begin{array}{l}\text { Number } \\
\text { If there are multiple files of the } \\
\text { same type this should be the } \\
\text { numerical indicator. i.e. "1" for } \\
\text { Video 1, "2" for Video 2, etc. }\end{array}$ & $\begin{array}{l}\text { Filename } \\
\text { This should be the name the file is } \\
\text { saved as when it is uploaded to our } \\
\text { system, and should include the file } \\
\text { extension. i.e.: Smith_ } \\
\text { Supplementary_Video_1.mov }\end{array}$ & $\begin{array}{l}\text { Legend or Descriptive } \\
\text { Caption } \\
\text { Describe the contents of the file }\end{array}$ \\
\hline Choose an item. & & & \\
\hline Choose an item. & & & \\
\hline Choose an item. & & & \\
\hline Choose an item. & &
\end{tabular}




\begin{tabular}{|l|l|l|l|}
\hline Choose an item. & & & \\
\hline Choose an item. & & & \\
\hline
\end{tabular}

Add rows as needed to accommodate the number of files.

\section{Source Data}

Complete the Inventory below for all Source Data files.

- Acceptable types of Source Data are:

o Statistical Source Data

- Plain Text (ASCII, TXT) or Excel formats only

- One file for each relevant Figure, containing all source data

o Full-length, unprocessed Gels or Blots

- JPG, TIF, or PDF formats only

- One file for each relevant Figure, containing all supporting blots and/or gels

\begin{tabular}{|l|l|l|}
\hline Figure & $\begin{array}{l}\text { Filename } \\
\text { This should be the name the file is saved as } \\
\text { when it is uploaded to our system, and } \\
\text { should include the file extension. i.e.: } \\
\text { Smith_SourceData_Fig1.xIs, or Smith_ } \\
\text { Unmodified_Gels_Fig1.pdf }\end{array}$ & $\begin{array}{l}\text { Data description } \\
\text { i.e.: Unprocessed Western Blots and/or gels, Statistical Source } \\
\text { Data, etc. }\end{array}$ \\
\hline Source Data Fig. 1 & & \\
\hline Source Data Fig. 2 & & \\
\hline Source Data Fig. 3 & & \\
\hline Source Data Fig. 4 & & \\
\hline Source Data Fig. 5 & & \\
\hline Source Data Fig. 6 & & \\
\hline Source Data Fig. 7 & & \\
\hline
\end{tabular}




\begin{tabular}{|l|l|l|}
\hline Source Data Fig. 8 & & \\
\hline Source Data Extended & & \\
Data Fig. 1 & & \\
\hline $\begin{array}{l}\text { Source Data Extended } \\
\text { Data Fig. 2 }\end{array}$ & & \\
\hline $\begin{array}{l}\text { Source Data Extended } \\
\text { Data Fig. 3 }\end{array}$ & & \\
\hline $\begin{array}{l}\text { Source Data Extended } \\
\text { Data Fig. 4 }\end{array}$ & & \\
\hline $\begin{array}{l}\text { Source Data Extended } \\
\text { Data Fig. 5 }\end{array}$ & & \\
\hline $\begin{array}{l}\text { Source Data Extended } \\
\text { Data Fig. 6 }\end{array}$ & & \\
\hline $\begin{array}{l}\text { Source Data Extended } \\
\text { Data Fig. 7 }\end{array}$ & & \\
\hline $\begin{array}{l}\text { Source Data Extended } \\
\text { Data Fig. 8 }\end{array}$ & & \\
\hline $\begin{array}{l}\text { Source Data Extended } \\
\text { Data Fig. 9 }\end{array}$ & & \\
\hline $\begin{array}{l}\text { Source Data Extended } \\
\text { Data Fig. 10 }\end{array}$ & & \\
\hline
\end{tabular}

